\title{
Persian Variations
}

\author{
Jean-Marc Castera ${ }^{1}$
}

Published online: 4 January 2016

(C) Kim Williams Books, Turin 2015

\begin{abstract}
The concept of variation is essential in geometric design. It is surprising that patterns very different may be variations of the same model. We define two families of pentagonal patterns with three kind of variations, and give some suggestions how to analyse these patterns and create in this style. We then search for self-similarity systems in a strict sense. Although from a systematic search, the two solutions proposed here can also generate some traditional 2-level patterns. In searching for subdivisions of the tiles into rhombuses, we found two solutions. Both can be compatible with the Binary Tiling (not with the Penrose Tiling). Then, using the concept of X-Tiles defined in a previous paper (Castera et al., http://castera.net/ entrelacs/public/articles/Flying_Patterns.pdf, 2011), we find new relationship between the two families of pentagonal patterns. In the last chapter we show and comment some examples taken from traditional architecture in Iran, and infer a selfsimilar system for pattern with interlaces from a 2-level tiling in Isfahan. This paper reflect the point of view of a pattern designer.
\end{abstract}

\section{Pentagonal Patterns}

\section{Terminology}

If everyone agrees more or less on what are the Pentagonal Patterns, it is not the case on what are their subfamilies, and how to name them. In this work I have to define subfamilies according to their components, name the tiles, and name some self-similar systems. For subfamilies, even if they are somehow similar to other defined by Bonner (2003, 2013); Cromwell (2009); Lee et al. (1987) and others,

Jean-Marc Castera

jm@castera.net

1 6, rue Alphand, 75013 Paris, France 
they do not have the same origin. That's why I have sometimes to use some specific names. Eventually, it is in the Iranian tradition that I found a classification of patterns close to the one proposed here. I will use it also, specially in the last part of this paper.

\section{Two Families of Pentagonal Patterns}

Let $\{\mathrm{S}\}$ be the "Starry Family" of pentagonal patterns, made of the set [S] of shapes derived from the decagonal star $\{10 / 3\}$, and $\{F\}$ the "Flower Family", whose main element is the standard 10-rosette. The first family is typically Persian, the second can be seen in any place of the historic world of Islam. We start talking about the Starry Family, and will meet the Flower Family in "Compatibility with the "XTiles", Binary Tiling and Link with the Flower Family".

\section{A Set of Tiles Generator of the Starry Family $\{S\}$}

Let it be [S]. All the angles in this set are multiples of $36^{\circ}$. The patterns made from it can be considered basic. They are usually subject to variations, that can generate new tiles which we consider as secondary. We will see in "Two Self Similarity Systems on $\{\mathrm{S}\}$ " that this set $[\mathrm{S}]$ have been defined from the search of selfsimilarity systems. The sub-set [S1] contains the 5 most common tiles, and have some remarkable properties.

First remark, there are two kind of tiles: the Positive Tiles, P1, P2,.., P5, and the Negative Tiles, N1, N2..., N6. When the pattern is colored like a chessboard, every Positive Tile is Black, while the others are White (or the contrary).

The additional lines, drawn on the Positive Tiles, are used only to make a link with the PIC method. Let them be the "Polygonal Lines".

The tile N1, which is the $\{10 / 3\}$ star, can be considered the mother of that set, although it is typically subject to variations. Those variations enrich the pattern, but can hide the main structure as well.

Note: That classification is not far from the one used by traditional artists in Iran (Mofid and Raieszadeh 1995; Shaarbaf et al. 1982), who consider 3 families of tiles: "Kond" (which correspond to [S1]), "Shol", and "Tond" (tiles of the second family, $\{F\}$, of pentagonal patterns. See components in Fig. 30). The set [S] correspond more or less to what they call "Kond and Shol" (Figs. 1, 2).

Rules for assembling the tiles It's obvious, but better to say: Tiles are put together in respect with the continuity of the edges. Thus, tiles of the same kind (Positive or Negative) are never connected by edges.

We could use only the Positive Tiles connected by vertexes (that's the simplest way to work with computer vector software). In that case, the shape of the holes are the shapes of the Negative Tiles.

First kind of variations When one tile is replaced by a set of tiles. Below, the variations on N1 and N6:

Each variation, except $\mathrm{N}_{2}$, generates secondary tiles. $\mathrm{N}_{2}$ include all components of [S1]. Sometimes it's not that obvious to recognize the hidden N1 in a complex pattern (This can be a good exercise). 


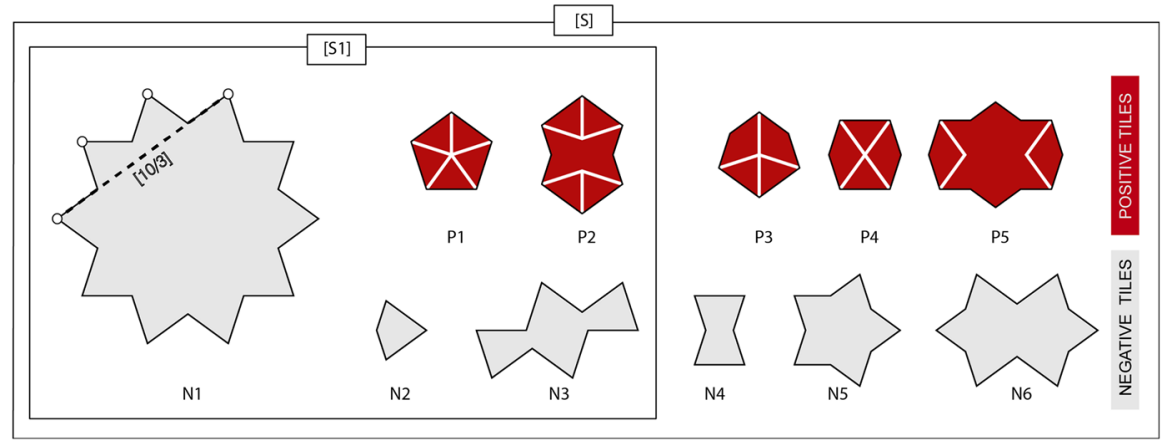

Fig. 1 The set of tiles [S] used in the "Starry" family of patterns, $\{\mathrm{S}\}$. In red the Positive Tiles (with addition of the "Polygonal Lines"). In light the Negative Tiles. N1 is "the mother" of this set. On the left, the sub-set [S1]

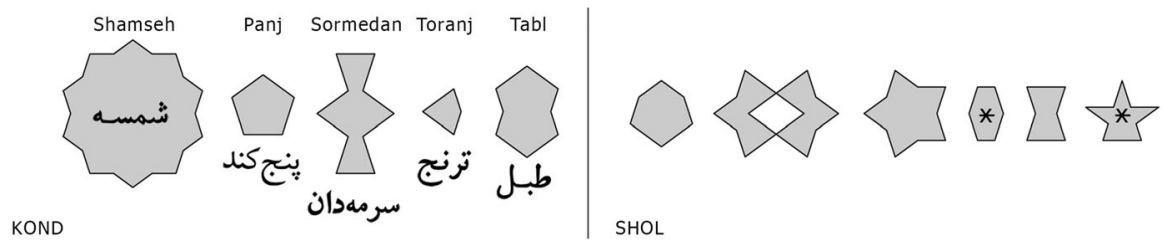

Fig. 2 "Kond" and "Shol" sets of tiles, from Iranian traditional classification. The Kond family correspond to [S1] even though the "mother" N1 is missing (because always decorated with a $\{10 / 2\}$

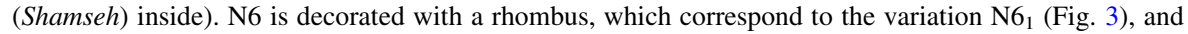
we have two extra Shol tiles (X) compare to [S]

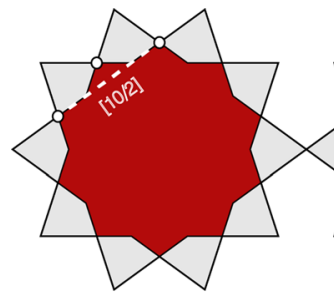

$\mathrm{N} 1$

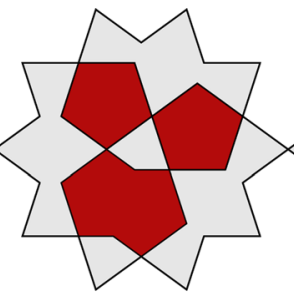

$\mathrm{N} 1$,

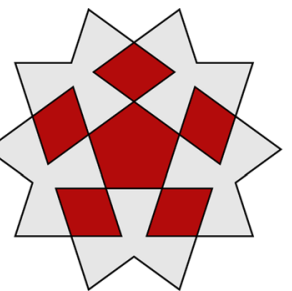

$\mathrm{N}_{3}$

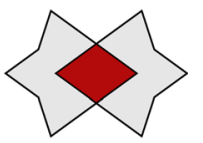

N6

Fig. 3 Variations on the "mother", N1. $\mathrm{N}_{2}$ contain every tiles of [S1], while $\mathrm{N} 1_{1}, \mathrm{~N} 13$ and $\mathrm{N6}_{1}$ generate new tiles

Second kind of variations when a connected set of tiles is replaced by another one.

The examples below (Fig. 4) introduces an element of the flower family $\{\mathrm{F}\}$, the famous "10-rosette", into the Starry Family $\{\mathrm{S}\}$.

Here two versions of this rosette, F1 and F2, are incorporated differently into a pattern of type $\{\mathrm{S}\}$. Although the scales are different, the proportions are the same (Fig. 5:with 4 edges equals, the hexagonal petals are as regular as possible). F1 can 


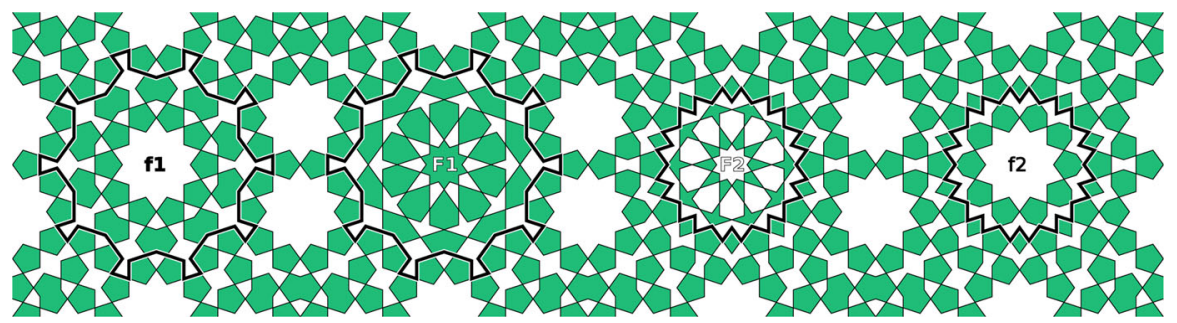

Fig. 4 Two different ways to incorporate the 10-rosette into the Starry family. F1 takes place of f1, F2 takes place of $\mathrm{f} 2$

Fig. 5 The 4 equal edges of the rosette's petals
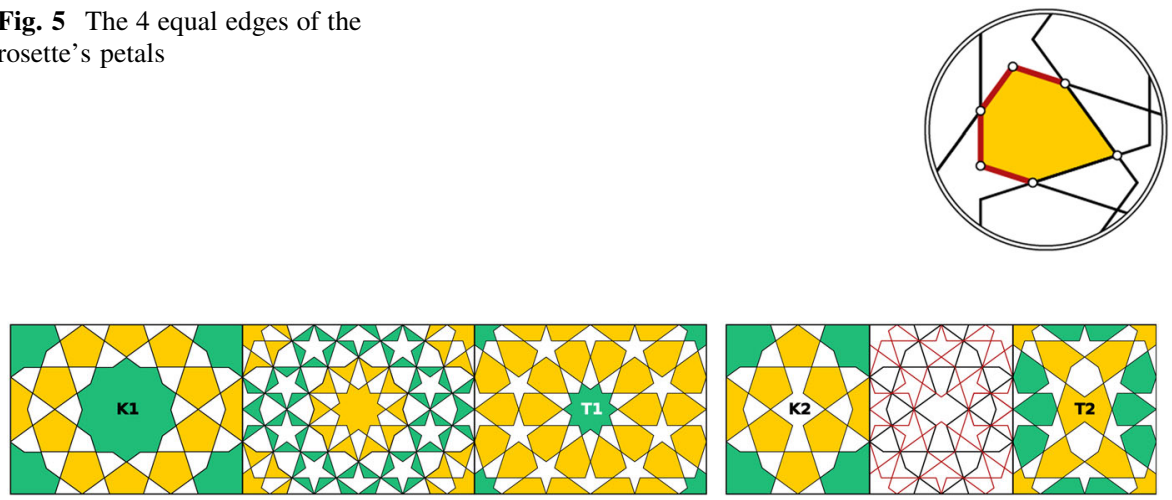

Fig. 6 Traditional transformations from "Kond" (K1, K2) to "Tond" patterns (T1, T2). The second case generates irregular rosettes at the corners

be seen on the famous Karatay panel in Konya (Fig. 13). The other one... in many places. Note the color inversion between F1 and F2 (Fig. 4).

Third kind of variations when a transformation is applied to the whole pattern. That definition include also the case of multilevel and self-similar patterns discussed in "Two Self Similarity Systems on $\{\mathrm{S}\}$ ".

The two examples (Fig. 6), from the Iranian tradition (Mofid and Raieszadeh 1995), show how to get patterns with rosettes from the two simplest Kond patterns $\mathrm{K} 1$ and K2. The first one (named "the mother of tilings" in Iran) gives way to the simplest pattern with 10-pointed rosettes, which can be seen everywhere in the world of Islam. The PIC method would give the same result in a different way. In the second case, the rosette has two types of petals.

Notice to the reader in order to always consider the patterns in their simplest form we do not use variations on N1 (the mother) in the following drawings, even though in the real world of traditional patterns of the starry family $\{\mathrm{S}\}$, the mother is always "pregnant" (always has something inside).

The reader is encouraged to design patterns in that way, and to introduce variations afterward.

But what are these Polygonal Lines, drawn on each Positive Tiles (Fig. 1)? Now, let's talk about the PIC method. 


\section{The Point of View from the PIC Method}

The polygon in contact (PIC) method proposed by Hankin (1925), improved by Kaplan and Bonner, can be used for constructing tilings, starting from an underlying polygonal pattern.

In the simplest - and more elegant—case, the polygons are equilateral. They are decorated by lines issued symmetrically from "Crossing Points" at the middle of each edge with a constant angle, running straight until they meet together. When the pattern is done, the polygonal lines are erased and it remains the actual tiling.

The qualities of the model have been widely praised in countless publications, so I would like to mention here some of its minor flaws. Of course, the reader unfamiliar with the PIC theory can skip this chapter.

1. In terms of pattern analysis, finding an underlying polygonal pattern do not answers to the question of how the later have been conceived and constructed. In term of creation, there is no evidence that it is easier to work with such polygons than directly with the actual tiles.

2. The decagon (Fig. 7-1) is always shown with a $\{10 / 2\}$ star, or "Shamseh", inside (variation $\mathrm{N}_{1}$ in Fig. 3). To be more consistent, it would be appropriate to add a rhomb in polygon (3) (variation $\mathrm{N}_{1}$ ).

By the way, and more important: the "mother" N1 is hidden.

3. The first set of equilateral polygons (1-6) is elegant. But, in order to work with more patterns, some non equilateral polygons are needed. Some crossing points are not at the middle of the edge (8), polygon (7) is "hybrid" (one edge having two crossing points on it), polygon (9) is undecorated and sometimes (not shown here) several decorations are used for a same polygon. May be effective, but not that much elegant.

4. The economy in terms of number of elements is poor. And if we consider that any pattern made with the set of tiles [S] could be made with only the Positive tiles connected by vertex, there is no economy at all.

Example: every pattern made from the set [S1] can be constructed with only the two Positive tiles of the set, while two or three polygons are needed with the PIC system.

5. Some patterns with elements from the two systems $\{\mathrm{S}\}$ and $\{\mathrm{F}\}$ cannot be produced-directly-from the PIC modules.

6. Some scholars find strong evidence in the use of PIC method by traditional Persian artists (Bonner 2003; Cromwell 2009, 2013; Lu and Steinhardt 2007;

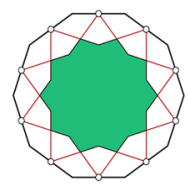

1

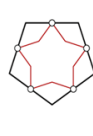

2

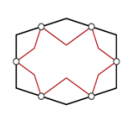

3

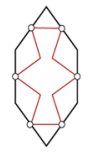

4

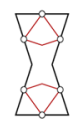

5

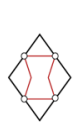

6

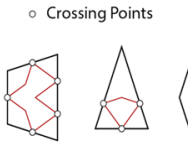

7

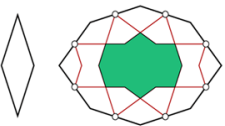

10

Fig. 7 Some polygons in use by Bonner, and their decoration in the case of "Median Design" (Bonner 2003, 2013) 
Makovicky 1992). That conventional view relies on a questionable interpretation of the Topkapi Scroll (Necipogglu 1995). Moreover, I did not find any mention of the PIC method in the Iranian books of tiling I know (Mofid and Raieszadeh 1995; Shaarbaf et al. 1982, other cited in Sarhangi 2012).

7. The relationship between the polygonal and the actual patterns is not necessarily a causal relationship, this is nothing more than a kind of duality. That's the reason why I have drawn the "Polygonal Lines" on the Positive tiles: In any pattern made from the set of tiles [S], those lines define automatically the "hidden" polygonal pattern, after erasing the orphan lines (Polygonal Lines having one vertex unconnected to another Polygonal Line).

\section{Example}

Let's start with the pattern previously designed for the variations with 10-rosettes (Figs. 8, 9, 10).

Note that:

1. The PIC method doesn't works properly for the connexion zone around the left rosette.

2. Five non equilateral polygons have to be define. Two of them are "hybrids" and the pairs of lines don't make always the same angle.

Anyway, some contemporary artists in the field are working with this technique, producing very good works (Bonner 2013; Pelletier et al. 2013). Several different methods can be used, each having its qualities and flaws. In another publication (Castera et al. 2011), I've imagined a morphogenesis of pentagonal patterns. I do not claim that it has been used at the historical period, but it works as well.

However, there is at least one case where this technique could be essential: the case of the 2-Points family, when there is not one but two connecting points on each edge (Bonner 2003, 2013; Kaplan 2005; Kaplan et al. 2002).

An interesting thing is the possibility to produce different patterns by changing the angle of the pairs of lines (What can be done interactively with Kaplan's Taprats software (Kaplan 2005, see also Bonner 2003, 2013).

Note that we could similarly define some polygonal lines on the negative tiles, like on the following image. This generates a new pattern, similar to those used for mashrabiyya (Figs. 11, 12).

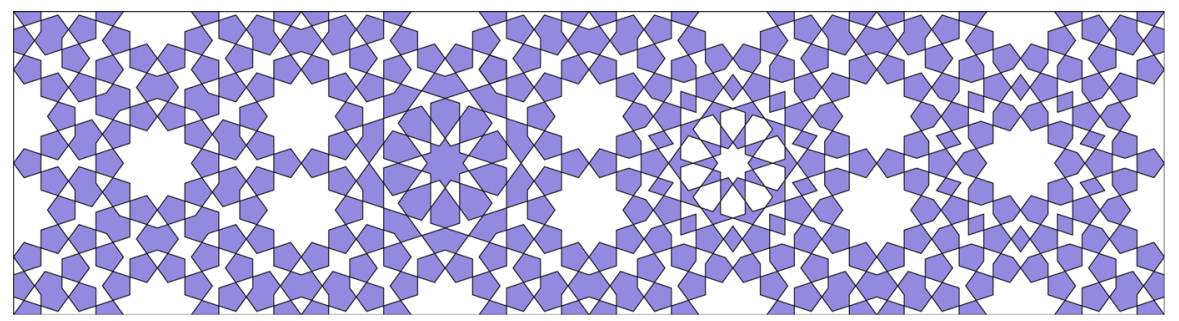

Fig. 8 The pattern (Made from the set [S1] with variations $\mathrm{N6}_{1}$ and rosettes) 


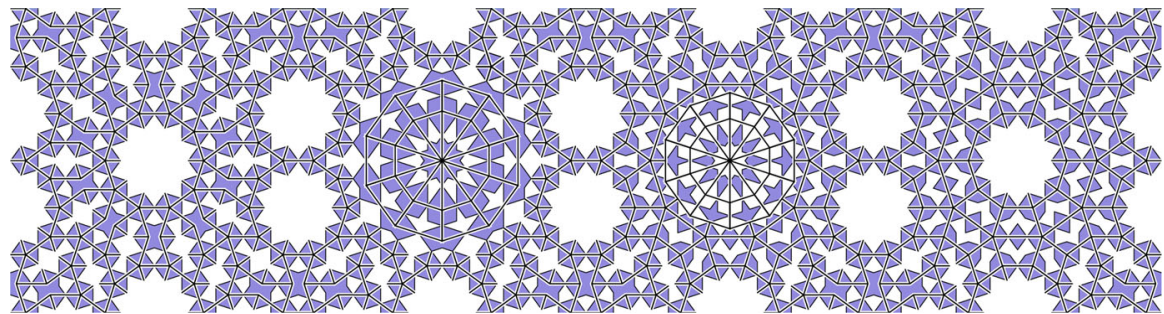

Fig. 9 With the Polygonal Lines drawn on each Positive Tile. Remove the orphan lines...

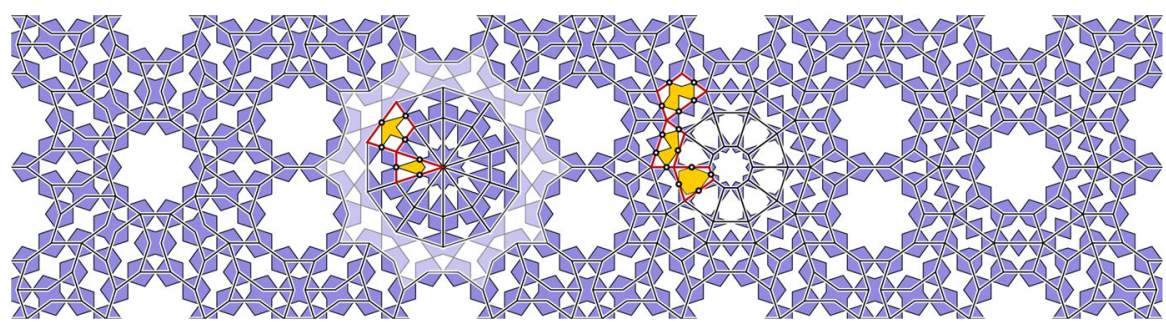

Fig. 10 ... And here is the polygonal pattern of the PIC method. The additional polygons are highlighted in bold

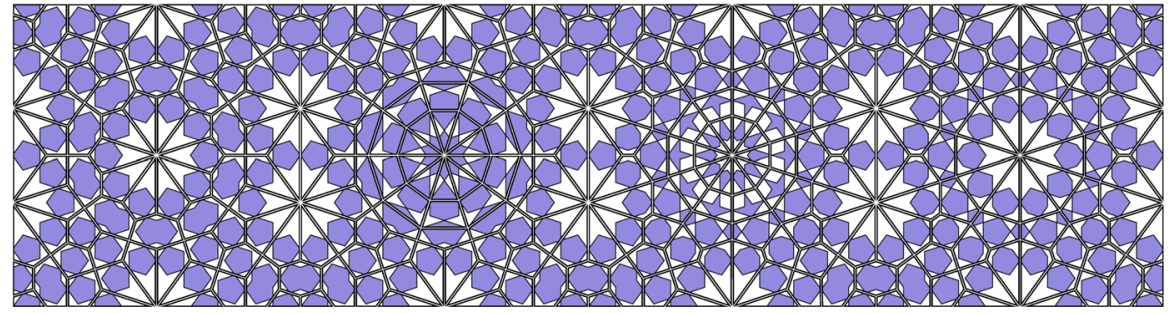

Fig. 11 Polygonal lines drawn on the negative tiles

The variation which consists to include the 10-rosette is an example of connexion between the two families $\{\mathrm{S}\}$ and $\{\mathrm{F}\}$ of Persian pentagonal patterns. We are going to meet other surprising connections in "Compatibility with the "X-Tiles", Binary Tiling and Link with the Flower Family".

\section{Suggestions for Analyzing a Pattern of the Starry Family $\{\mathbf{S}\}$}

The key words are: simplification and main lines. After analyzing the symmetries, try to find the other main construction lines (like what you do when sketching from life). Then try to identify what is not essential, where are the variations, in order to get the simplest basic pattern. ${ }^{1}$

\footnotetext{
${ }^{1}$ I strongly recommend also the reading of every publication from Antony Lee, Peter Cromwell (available on http://www.liv.ac.uk/ spmr02/islamic/), the new book of Jay Bonner: Islamic Geometric Patterns: Their Historical Development and Traditional Methods of Derivation, once it's published, C. Kaplan's thesis, and to experiment the software "Taprats", from C. Kaplan as well.
} 


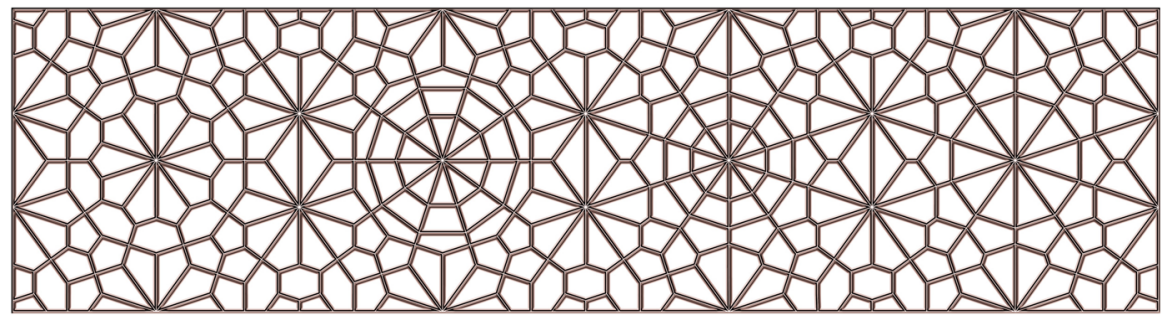

Fig. 12 Same pattern with only the negative polygonal lines

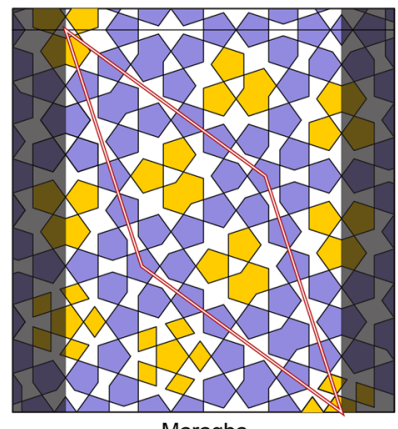

Maragha

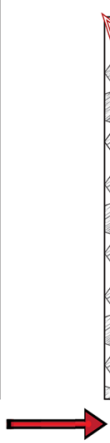

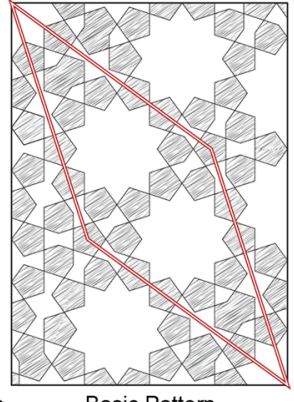

Basic Pattern

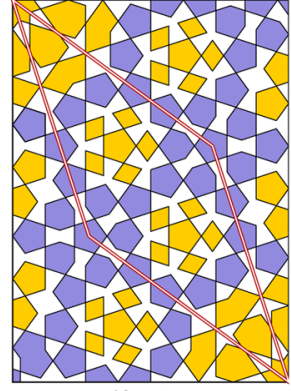

Karatay

Fig. 13 Once all variations (colored in yellow) have been removed, these two famous patterns can be seen as different interpretations from the same basic design (middle). Left Maragha blue tomb, Iran. Right Karatay medersa, Konya, Turkey

As an example, let's consider two famous patterns: One from the Maragha blue tomb, Iran, the other one from Karatay medersa, Konya, Turkey (Fig. 13).

I've drawn the entire Maragha panel (left), and reduced the other to 1/4 (right). The variations $\left(\mathrm{N}_{1}, \mathrm{~N}_{2}, \mathrm{~N}_{3}\right.$ and rosettes) are colored in grey. Both units have the same structure: a great lozenge on the diagonal.

Then, after reducing the patterns to their simplest expression (removing all variations), it remains exactly the same basic pattern (which comes from the 4th generation in my morphogenesis process, see Castera et al. 2011). That surprising fact would be difficult to see without any simplification.

\section{Experimentation}

The kind of representation needed differs according to what you are doing with patterns: Analyse, conception or making.

Conception It makes sense to work directly with the tiles. Although there is no evidence, I can imagine that historical designers could have used paper tiles models to aid in the creation. In Morocco, some masters use directly the Zellij tiles (Paccard et al. 1980, vol. 1, p. 396), or different techniques (Castera 1996) (but not the PIC method). Moreover the artists, as the mathematicians, can have a right reasoning from a bad figure.

In terms of making, it depend of the technique. If woodwork or plaster needs a drawing or a stencil in real size, this is not the case with the ceramic technique, 
where the tiles are cut individually from a template, and put together afterward on a plane surface where only the symmetry lines are drawn. The template for each kind of tile has to be as perfect as possible, but this needs nothing more than elementary geometry. When a drawing is needed, it don't need to be geometrically perfect. For example, no sophisticated geometry is needed to divide with a compass a circle into 5 or 7 sufficiently equal parts: simply try and correct.

The above photos show the first game I did to study a sub-set of $\{\mathrm{S}\}$ by experience. There are only 3 Positive tiles (A new version includes all the tiles of the set [S]), with the Polygonal Lines engraved. The corresponding polygonal pattern appears naturally when we play with the puzzle, but here this is not the cause but the consequence. With this set of tiles, we can make non-periodic patterns as well as periodicals (Fig. 14).

\section{Two Self Similarity Systems on $\{S\}$}

In this research to self-similarity systems I consider the actual tiles of the patterns, never the associated polygons.

I consider the simplest version of the patterns, without variations. Thus, "the mother" of the tiles, N1, will be seen in its pure beauty...

Although they come from a systematic study, and not from the historical patterns, it comes out that the first solution is related to the 2-level patterns of the Topkapi

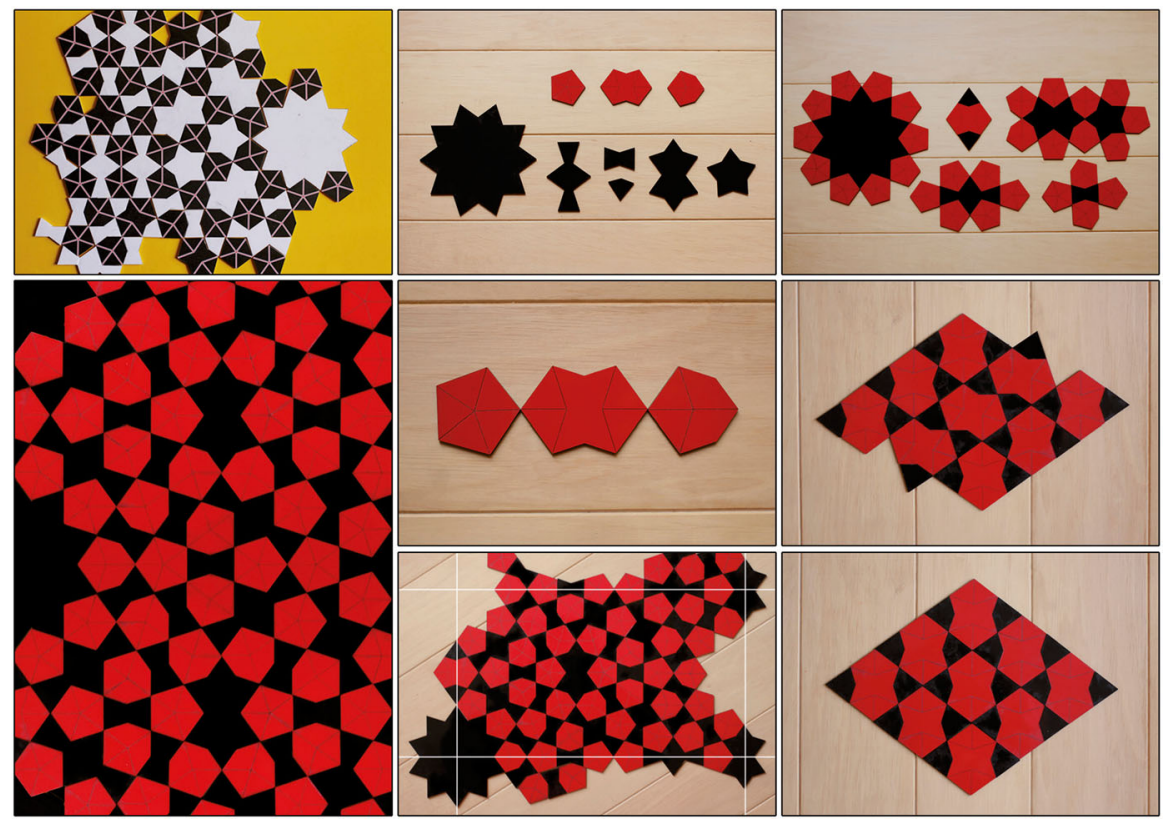

Fig. 14 Top-right Connected by vertices, respecting the continuity of the edges, the Positive Tiles generate the Negative Tiles (in black). So, we basically could use only the Positive Tiles (in red)... with the holes in between 


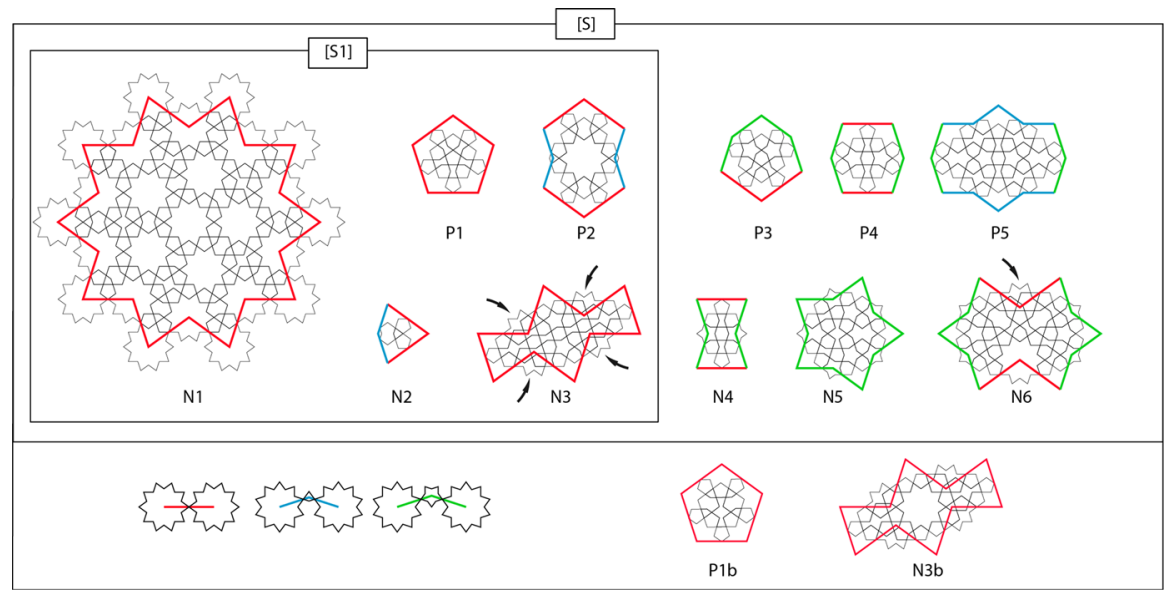

Fig. 15 First system of self-similarity, "in the style of the Topkapi Scroll”. It comes from the mapping of the 3 different kind of edges (bottom, left). Each tile of the set [S] is mapped by reduced tiles of the same set. P1b and N3b are alternative solutions for the tiles P1 and N3

Scroll (Necipogglu 1995), and the second to many patterns that can be seen in Isfahan (see "Multilevel Patterns").

Each solution is characterized by the scale ratio, $\mathrm{k}$, between two successive levels (so $\mathrm{k}>1$ ).

I could not get a solution between the two which we are going to discuss here, and haven't searched that much on solutions with an upper scale ratio... until I met a special pattern in Isfahan ("Self-Similarity with Interlaces").

\section{Definition}

We call self-similar pattern (in the strict sense) a pattern made from a limited set of tiles, with the following properties:

1. Each tile of the set can be patterned with tiles of the same set at a reduced scale.

2. Applying these transformations to each tile of the pattern produces a new pattern without any gap or overlapping, ${ }^{2}$ so the process could go infinitely, level by level.

3. Any level can be mapped onto the next level (not onto itself because we are considering real patterns, which are finite).

Here we are searching for solutions in which each different shape is associated to one single transformation rule. These rules are named "Inflation Rules".

In terms of visualization, 2 levels are enough, under the condition that the transformations are defined for every tile at the first level (with no extra tile at the second level).

2 To be exact, we would have to add a condition: either to accept a perfect superposition of some second level tiles along the edges of the first level, or to use a solution like in Fig. 16, with cut tiles. 
According to this definition, no drawing of the Topkapi scroll is self-similar. I have not yet recognized a self-similar traditional pentagonal pattern, but only 2-level patterns (Necipogglu 1995 pl. 28, 29, 31, 32, 34, and "Examples in the Iranian traditional Architecture" of this paper). Some may be very close, like at Darb-e Imam, Isfahan, but there is always something missing.

Peter Cromwell (2013) have recognized a scale-invariant design in the hexagonal family of Indian style.

Peter Lu and Steinhardt (2007) state that the famous Darb-e Imam shrine pattern (Fig. 57) is self-similar, according to the PIC point of view. But the first level pattern would be made from 2 polygons, while the second level needs one more (the hexagon). One inflation rule is missing, and the first level -which is periodic- cannot be mapped onto the second.

\section{Method}

We first look for a way to pattern the edges of each shape with reduced tiles, and then try to fill the interiors.

\section{First Solution, Minimal Scaling (Ratio Maximum)}

The scale ratio is equal to $3+\sqrt{ } 5$.

There is a strong similarity to the Topkapi Scroll 2-level patterns.

It works for any pattern made from the set [S]. In fact, this set has been defined from this process (Fig. 15).

This mapping of the set of tiles [S] is strictly self-similar, even if we use the mapping P1b in place of P1 or N3b in place of N3. But not the both: in this case, the sub-set [S1] himself would be self-similar. You can imagine the consequences...

Take care to the mapping of N3, with its "little wings under the armpits" (arrows). When connected to a Positive Tile those wings break the usual star centered on the vertex. So we will not have to define different configurations for each positive tile. Same thing with N3b and N6.

Could we make a puzzle in real from that set? Certainly (Fig. 16), but the shapes are a little bit strange, as you can see.

\section{Second Solution, "Isfahan Style"}

This solution do not works for the complete set [S], but only for the sub-set [S1].

The scale ratio is equal to $4+2 \sqrt{ } 5$.

There is a strong similarity to some 2-level patterns that can be seen in Isfahan, Iran (Fig. 17).

At the left, some other options (not exhaustive) for paving the tiles P1, P2 and N3. Again, the tile N3 needs "little wings under the armpits" because it cannot be a complete star here.

If we start the inflation process from any tile, the second level will always include the complete set [S1]. So, at the third level every transformation rule will have been 


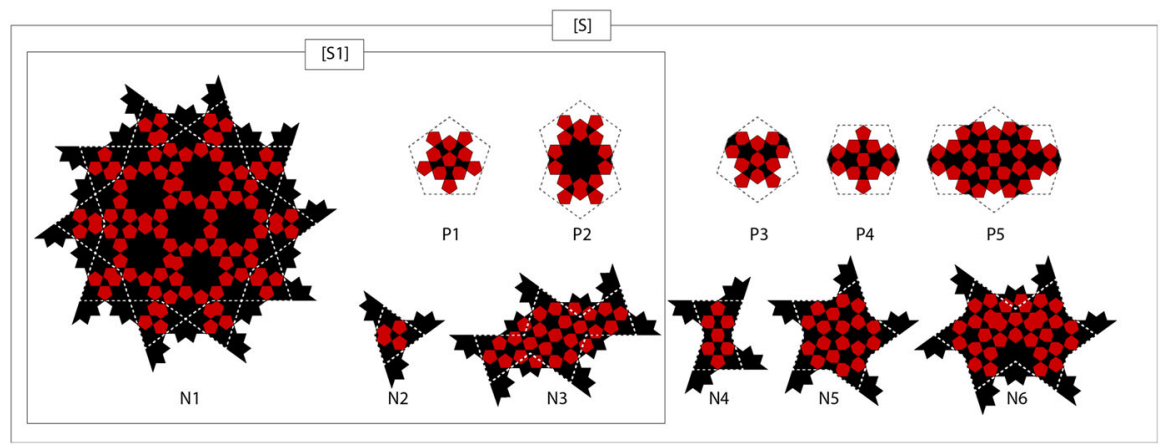

Fig. 16 Proposal for the making of real multilevel tiles that can be put together without any problem of overlapping on the edges

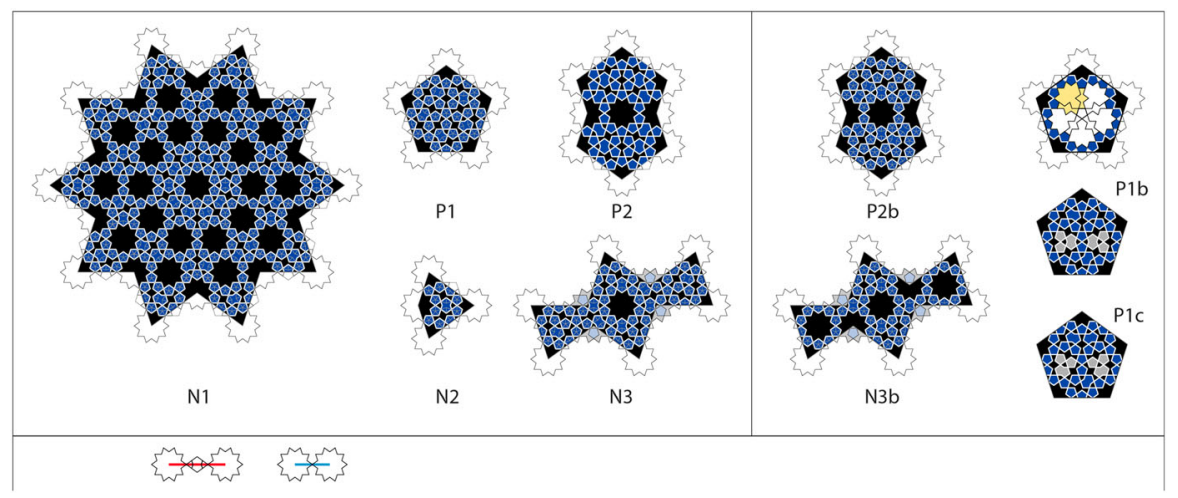

Fig. 17 Second system of self-similarity, "in the style of Isfahan". It works only for the subset [S1]. It comes from the mapping of the 2 different kind of edges (bottom). Each tile of [S1] is mapped by reduced tiles of the same set. Right P1b, P1c, P2b and N3b are alternative solutions for the tiles P1, P2 and N3 (not exhaustive)

defined and the pattern will be self-similar in the strict sense. At infinitum, it becomes a kind of 2D-quasicrystal.

The second-level elements are, for each tile:

With the first solution

$\mathbf{P} 1=>\mathrm{P} 1, \mathrm{P} 3, \mathrm{~N} 1, \mathrm{~N} 4 ; \mathbf{P} 2=>\mathrm{P} 1, \mathrm{P} 2, \mathrm{~N} 1, \mathrm{~N} 2 ; \mathbf{P 3}=>\mathrm{P} 1, \mathrm{P} 3, \mathrm{~N} 1, \mathrm{~N} 4, \mathrm{~N} 5 ;$ $\mathbf{P 4}=>\mathrm{P} 1, \mathrm{P} 3, \mathrm{P} 4, \mathrm{~N} 1, \mathrm{~N} 4, \mathrm{~N} 5 ; \mathbf{P} 5=>\mathrm{P} 1, \mathrm{P} 3, \mathrm{P} 4, \mathrm{~N} 1, \mathrm{~N} 3, \mathrm{~N} 4, \mathrm{~N} 5, \mathrm{~N} 6$; N1 => P1, $\mathrm{P} 2, \mathrm{~N} 1, \mathrm{~N} 2, \mathrm{~N} 3 ; \mathbf{N} 2=>\mathrm{P} 1, \mathrm{P} 2, \mathrm{~N} 1, \mathrm{~N} 2 ; \mathbf{N} 3=>\mathrm{P} 1, \mathrm{P} 3, \mathrm{P} 4, \mathrm{~N} 1, \mathrm{~N} 3, \mathrm{~N} 4, \mathrm{~N} 5 ;$ $\mathbf{N} 4=>\mathrm{P} 1, \mathrm{P} 3, \mathrm{P} 4, \mathrm{~N} 1, \mathrm{~N} 4$, ; N5 = P $1, \mathrm{P} 3, \mathrm{~N} 1, \mathrm{~N} 3, \mathrm{~N} 4, \mathrm{~N} 5, \mathrm{~N} 6 ; \mathbf{N 6}=>\mathrm{P} 1, \mathrm{P} 2, \mathrm{P} 3$, P5, N1, N2, N3, N4, N5.

Depending of the starting tile, we get the complete set [S] decorated at level 3 (from P5 or N6), 4, or 5 (from P2, N1 or N2).

With the second solution, we have the complete set [S1] decorated at the third level for each tile. 


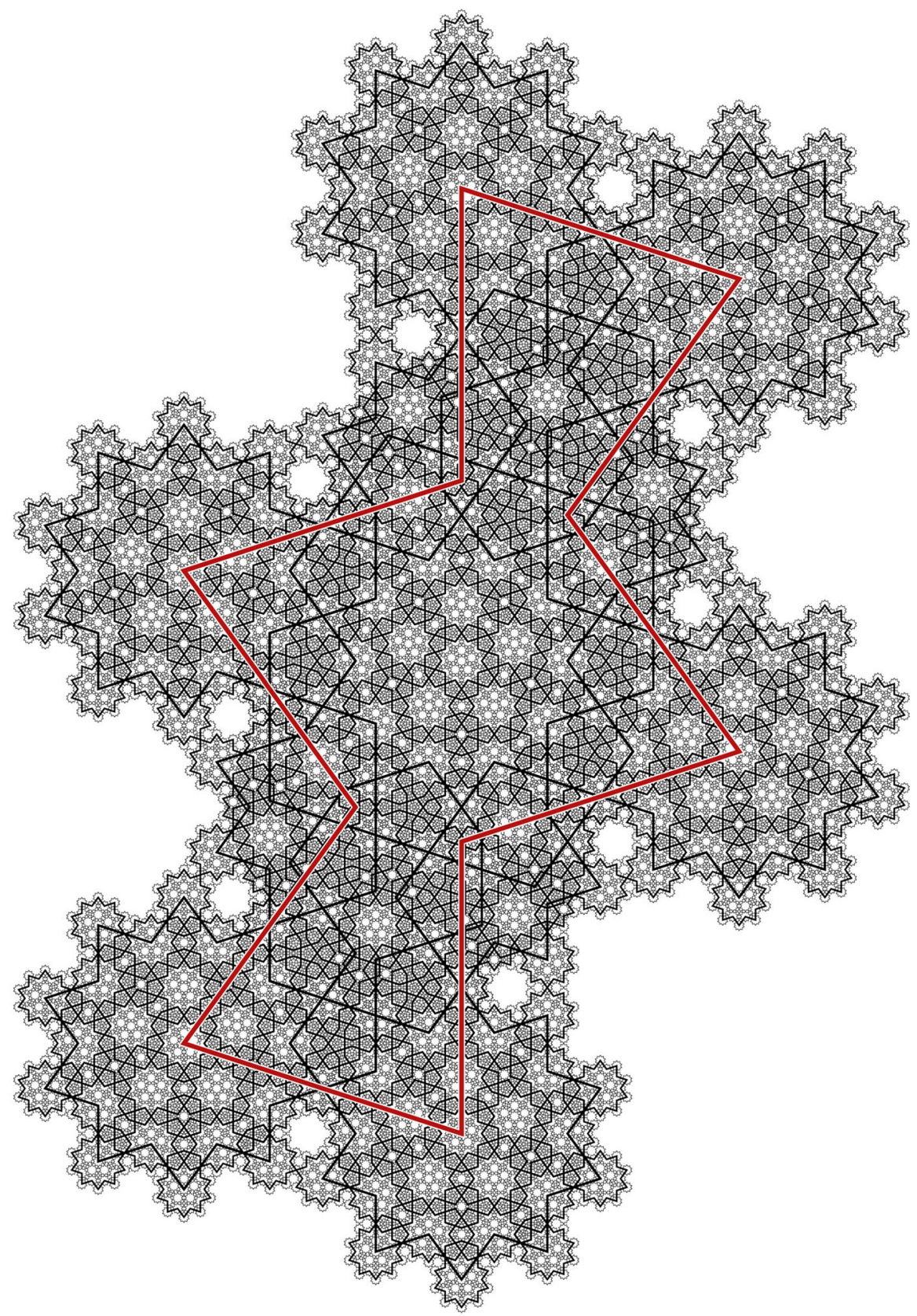

Fig. 18 The first system of self-similarity is applied to the tile N3 with the mapping option N3b. Four levels are drawn on the figure. The two first levels belong to the family $\{$ S1 $\}$ (tiles P1, P2, N1, N2, N3). P3 appears at the 3rd step, N5 and N6 at the 4th. Two steps are missing for having the complete set of mapped tiles. That slow process is due to the use of N3b in place of N3 


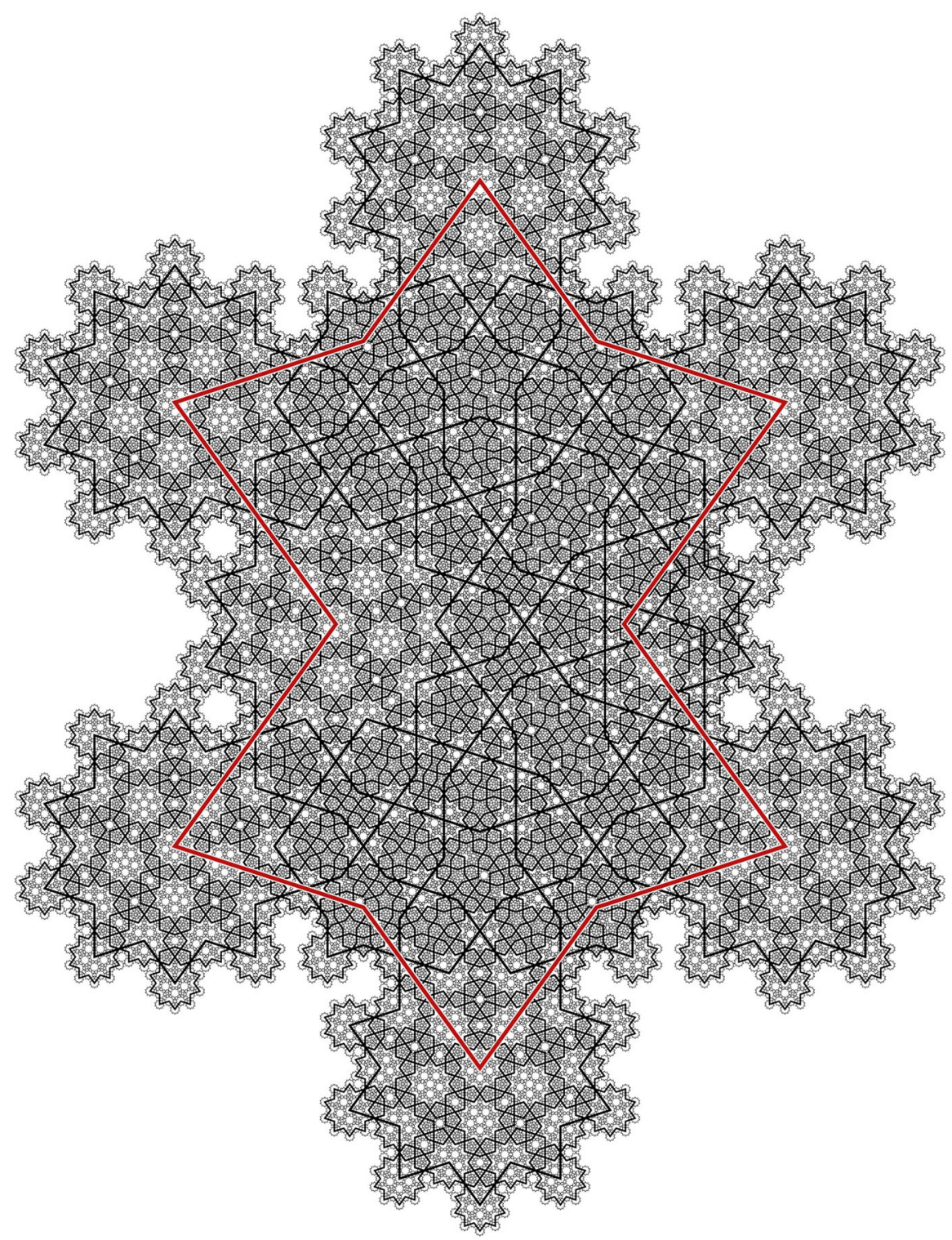

Fig. 19 Starting from the tile N6, we get the complete set of mapped tiles at the 4th level

Next pages, three generations of the first system are applied from N3b (Fig. 18) and N6 (Fig. 19), so we get a 4-level pattern. Note that, to avoid falling into the "[S1]-attractor" we have not used P1b but P1.

Two generations of the second system are applied from the tile P2 in (Fig. 20). The next figure (Fig. 21) shows the same pattern drawn with only the Negative Polygonal Lines defined above ("The Point of View from the PIC Method", Fig. 12). 


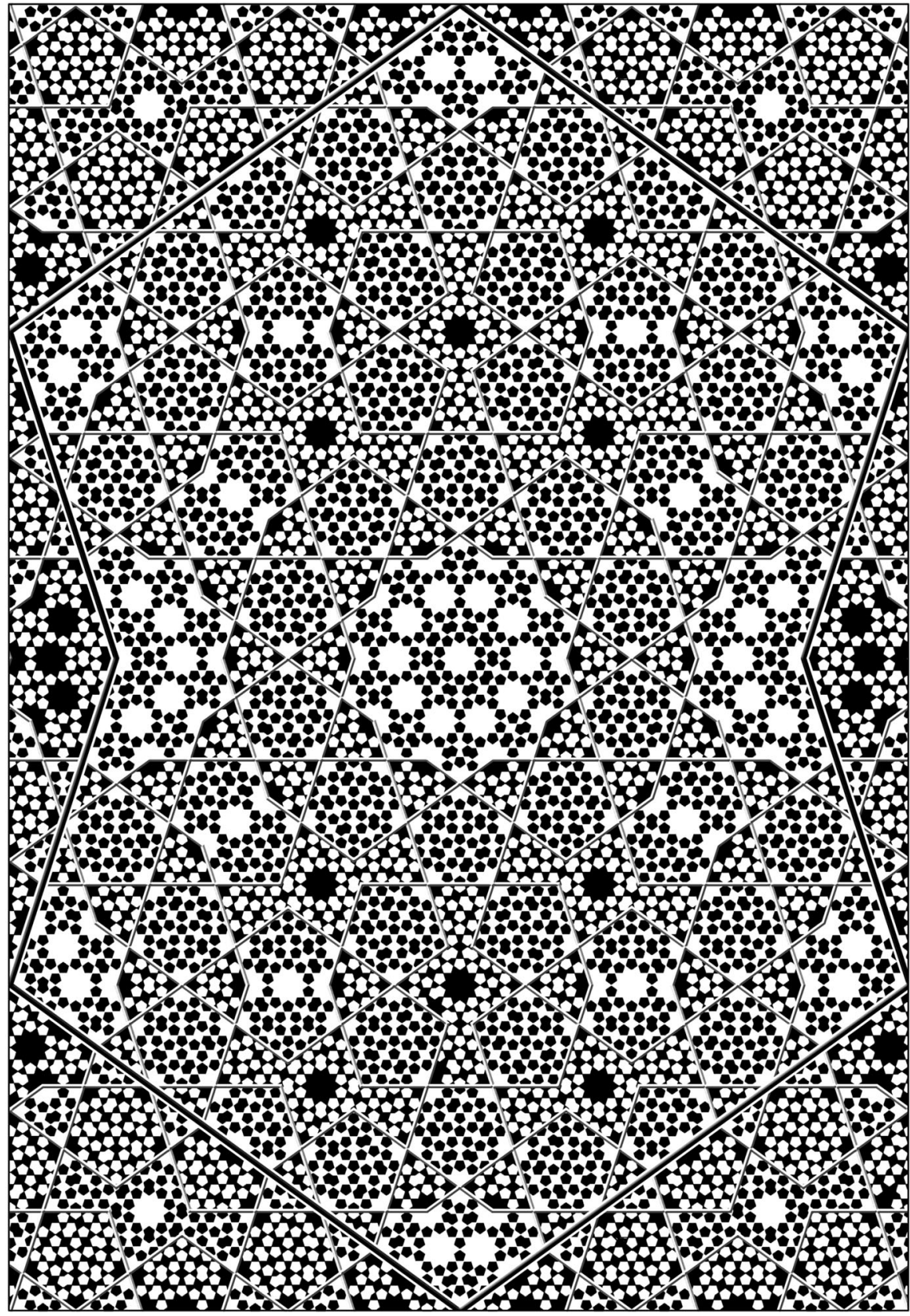

Fig. 20 The second process of inflation (Isfahan Style) applied twice from the tile P2. The pattern has been incorporated into a rectangular frame, so it could be repeated after some local adjustments along the vertical edges. We have used color inversion in order to distinguish the tiles at each level, although using only two colors 


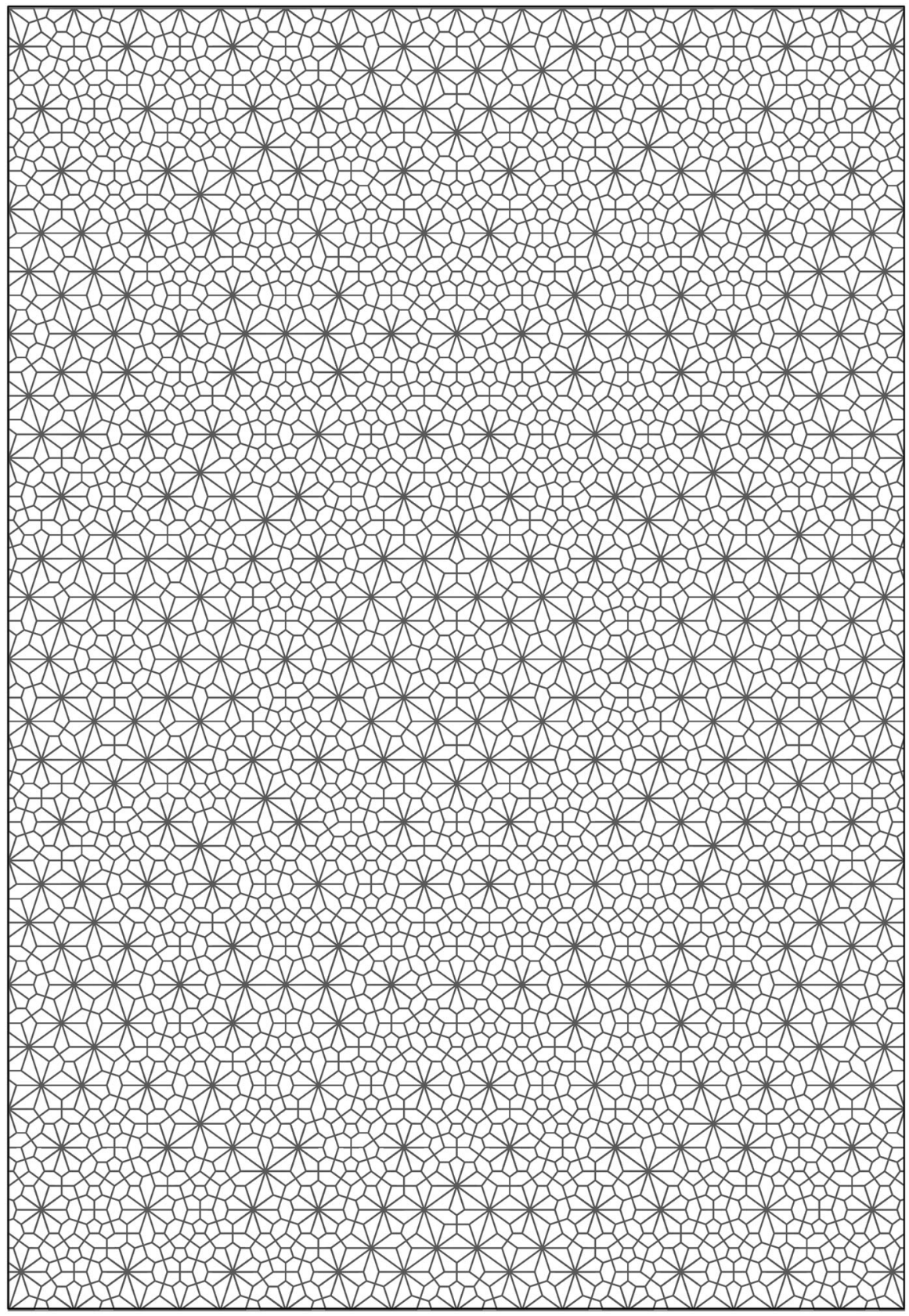

Fig. 21 Here is the same pattern with only the polygonal lines drawn on the Negatives Tiles, as defined previously. Mashrabiyya style? 


\section{Tiles Decomposition into "Penta-Rhombs"}

In a previous work on the octagonal system (Castera 2008) it came out that each tile of a certain sub-set of the elementary elements of Zellij can be made from the two components of an Ammann tiling, which are a square and a $45^{\circ}$ rhomb. Now the question is: could we define such a process for the pentagonal family? More precisely, for the set $[\mathrm{S}]$ ?

I've found 2 solutions. The first works only for the sub-set [S1] while the second works for the whole set $[\mathrm{S}] \ldots$ with some special conditions.

Obviously, the rhombs in the pentagonal system have to be the two famous used in a Penrose pattern. Let them be the "Penta-Rhombs" (Fig. 22).

\section{A First Solution}

The method starts by searching for different ways to map the edges of the tiles with the short (d) and long (D) diagonal of the rhombs. Then, trying to extend the mapping to the whole tile (Fig. 23).

This solution can be define by the mapping of the edge of the pentagon: $2 \mathrm{D}-1 \mathrm{~d}$ (long diagonal of 2 and short diagonal of 1). Unfortunately, it doesn't works for every tile of [S], but it works perfectly for [S1].

\section{A Second Solution}

That one works better (Figs. 24, 26), but the mapping needs some extra pentagons for the tiles P5 and N5.

Bottom right, we can see that there is no way to solve the problem, because the pentagon cannot be entirely broken into rhombs.

Anyway, the mapping works perfectly for the set [S1].

Note the two options for the same area, Rose 1 and Rose 2. On P1 and N3 we have chosen the second option. The reason will be given in the next chapter.

A slight variation of this solution is given on "The X-Tiles in historic patterns" (Fig. 53).

Next page, the two mappings are applied on the same pattern, the one we have previously used to illustrate the second system of self-similarity (Figs. 25, 26).
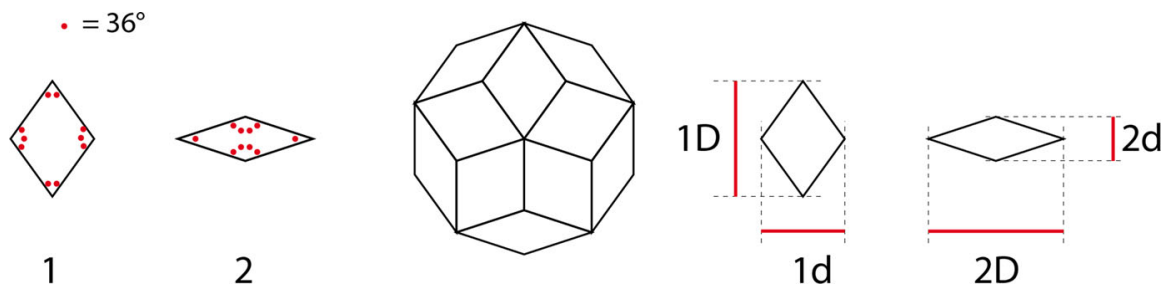

Fig. 22 The two Penta-Rhombs mapping the decagon (center), with indication of the angles (left), and of the lengths (right). For each tile ( 1 and 2 ) we note $\mathrm{d}$ the short diagonal and D the large one 


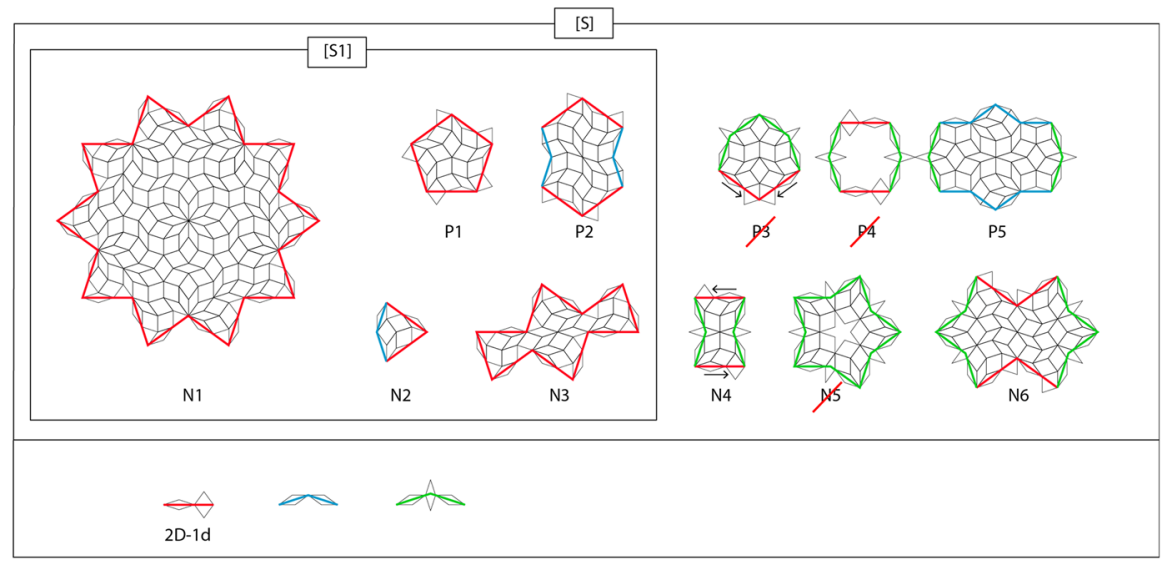

Fig. 23 First solution for mapping the tiles with the Penta-Rhombs. It cannot works for P3, P4 and N5. Bottom, the mapping of the three different edges

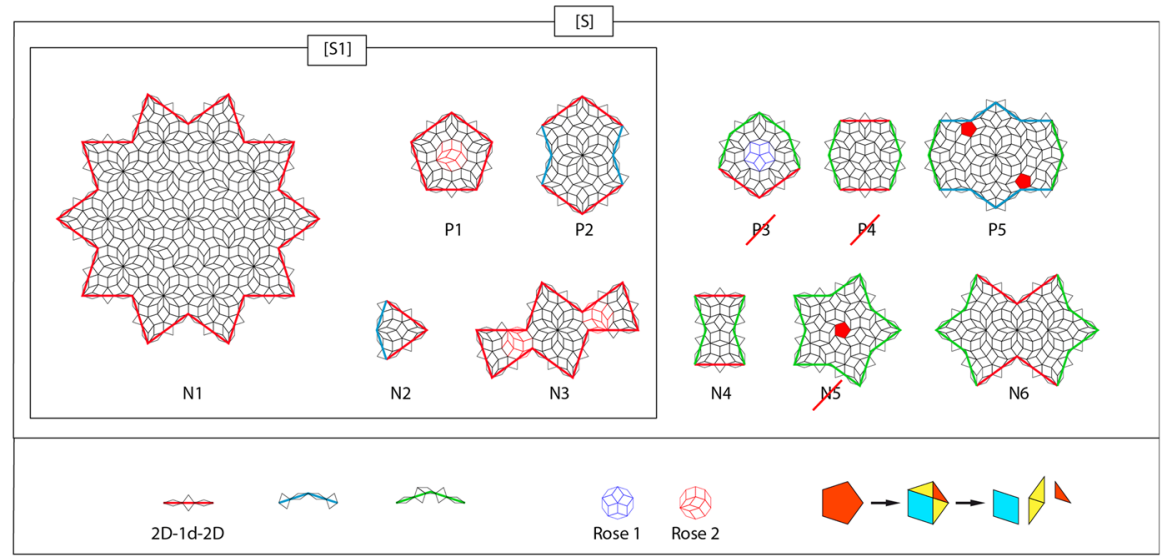

Fig. 24 Second solution for mapping the tiles with the Penta-Rhombs. Bottom left, the mapping of the edges

\section{Compatibility with the "X-Tiles", Binary Tiling and Link with the Flower Family}

We have seen that any pattern made from [S1] can give way to a pattern made of the two Penta-Rhombs. Is that pattern a Penrose Tiling? In fact, while mapping the tiles we were thinking about the "X-Tiles" previously discovered (Castera et al. 2011). Though they are the same rhombs as in Penrose's patterns, they don't follow the same matching rules. The matching rules for the $\mathrm{X}$-Tiles are the same as the ones in use for the "Binary Tiling" as defined in quadibloc.com/math/pen02.htm. They can generate non-periodic patterns and, unlike Penrose's matching rules, periodic patterns as well (so, they are not aperiodic). 


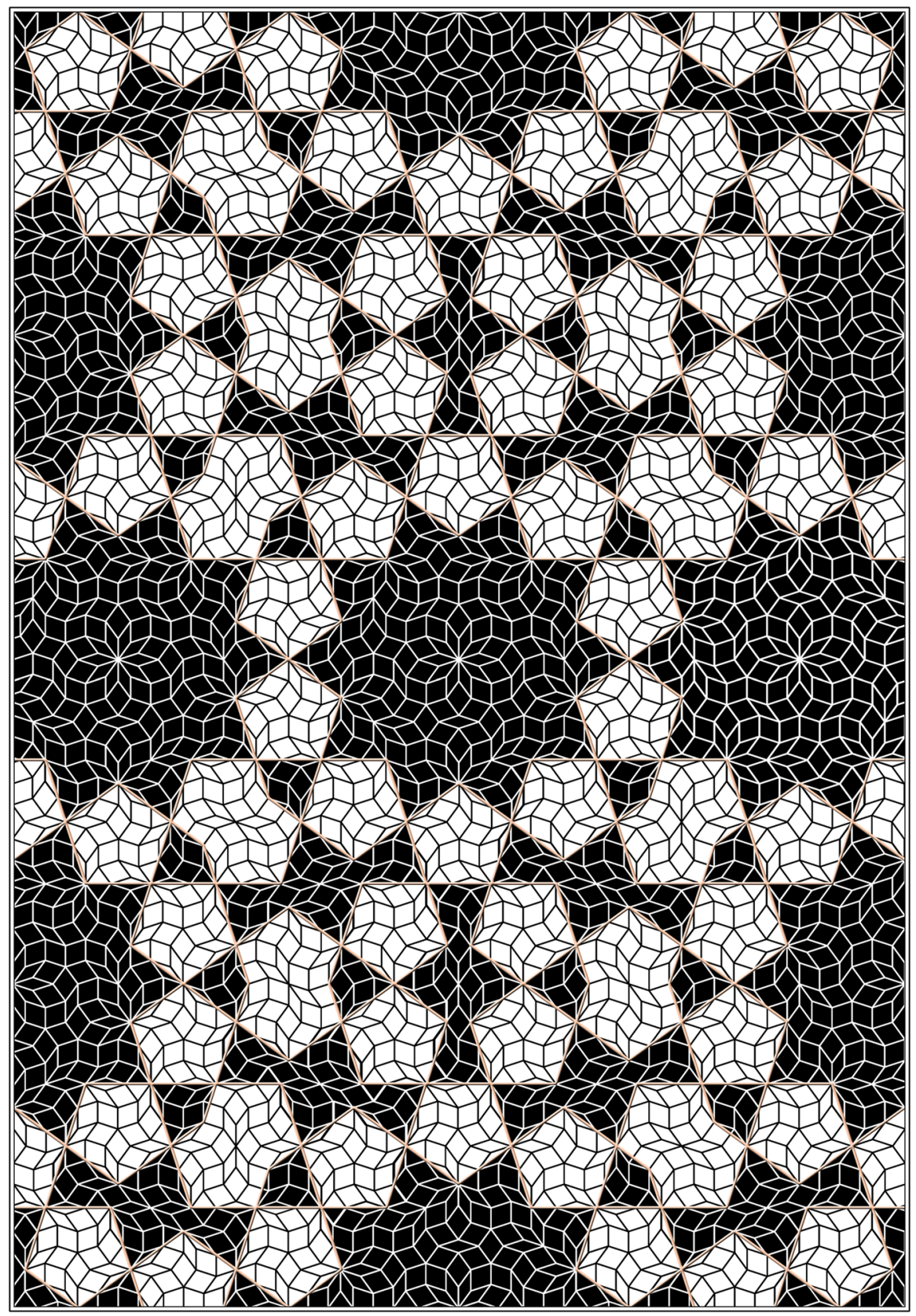

Fig. 25 The first solution of mapping the tiles with Penta-Rhombs, applied on a pattern made of tiles from $[\mathrm{S} 1]$ 


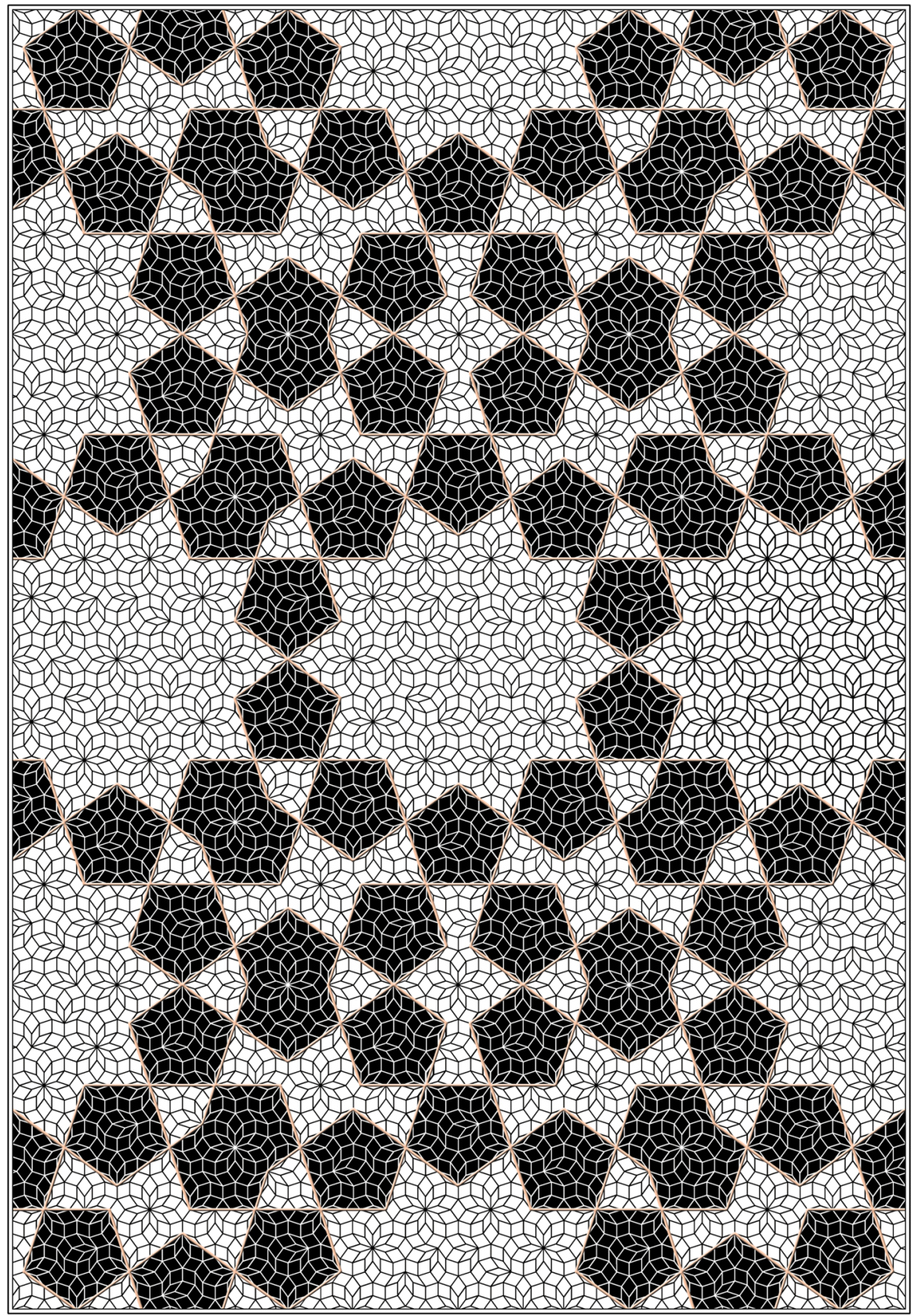

Fig. 26 The second solution of mapping the tiles with Penta-Rhombs, applied on a pattern made of tiles from $[\mathrm{S} 1]$ 


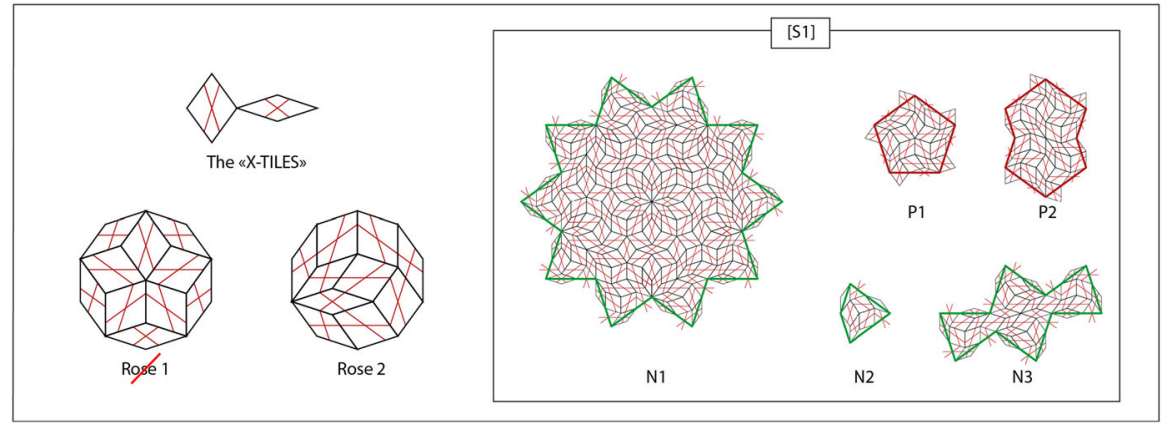

Fig. 27 Left-up, the "X-Tiles", with the "X-lines" in light. Bottom, the two mappings of the Rose. Only the second is compatible with the matching rules for the X-Tiles (respecting the continuity of the $\mathrm{X}$-Lines). Right, we can see that the mapping of the set [S1] is compatible with the X-tiles

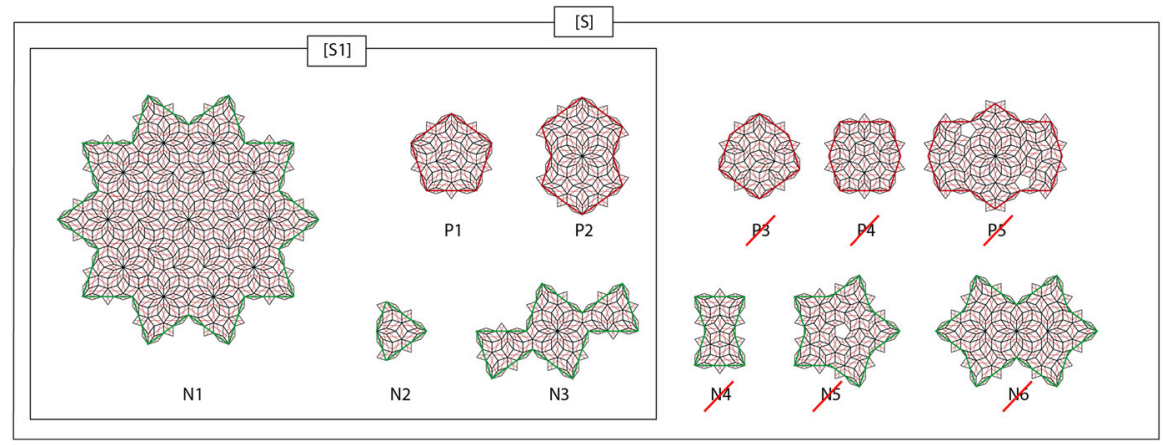

Fig. 28 The X-Lines drawn on each Penta-Rhomb of the second solution of mapping shown in Fig. 24

We are going to see that the two mappings proposed before are compatible with the X-Tiles. They generate patterns that belong to $\{F\}$, the Flower Family of pentagonal patterns.

A surprising result, isn't it?

Application of X-lines on the first mapping solution (Fig. 27).

Application of $\mathbf{X}$-lines on the second mapping solution (Fig. 28).

The whole set [S] is not compatible, but [S1] works perfectly.

Figure 29 shows the set $[\mathrm{X}]$ of all the tiles that can be drawn from the X-Lines. They belong to the traditional Iranian style "Tond" (Fig. 30). As for the tiles of [S], we can distinguish Positives and Negatives tiles. Any pattern made with these 9 tiles (There are a lot) can be made from the X-Tiles.

Next pages, some illustrations.

Again, we are starting from the same pattern. The Figs. 31 and 33 comes from the first mapping system after replacing each rhomb by the associated "X-lines", and from the second on Figs. 32 and 34. 


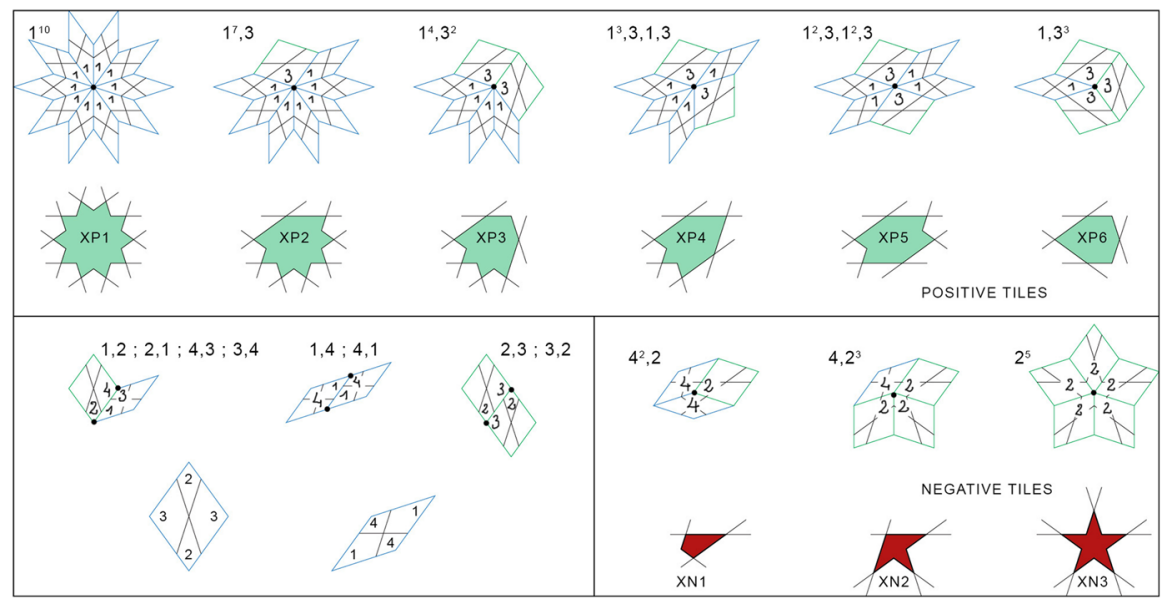

Fig. 29 All possible arrangements of X-tiles around a vertex. In color, the associated tiles drawn by the $\mathrm{X}$-Lines. The numbers $1,2 \ldots 4$ are notation for the angles (multiples of $36^{\circ}$ ). Bottom left the impossible combinations. Image taken from (Castera et al. 2011)

TOND
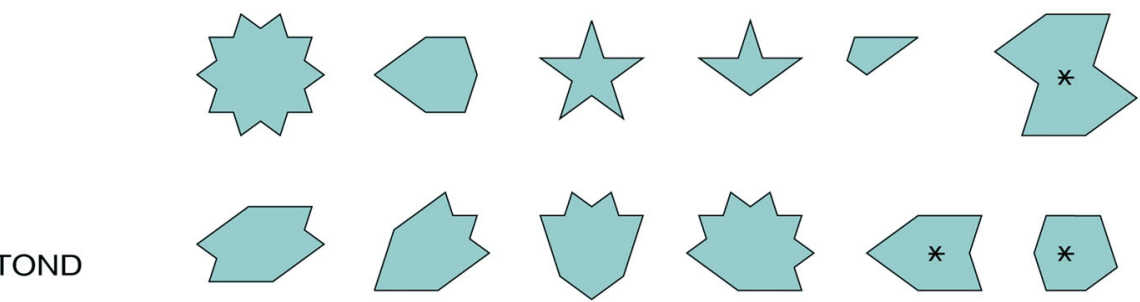

Fig. 30 The Iranian traditional set of tiles "Tond" include 3 extra tiles (X) compared to the set of tiles made from the X-tiles. Note that in this set the $\{10 / 3\}$ star is undecorated

On the two last figures we have highlighted the tiles along the edges of the original pattern. You can see that it works perfectly with the second mapping. Even though it looks similar to the skeletons in use for the octagonal system in Morocco (Castera 1996), it does not follow the same logic.

\section{Examples in the Iranian traditional Architecture}

\section{Starry Family $\{\mathbf{S}\}$}

Variations on the "mother of tilings" K1, the simplest Kond pattern (Figs. 35, 36, $37)$.

\section{Flower Family $\{\mathbf{F}\}$}

Variations on the simplest Tond pattern, T1. 


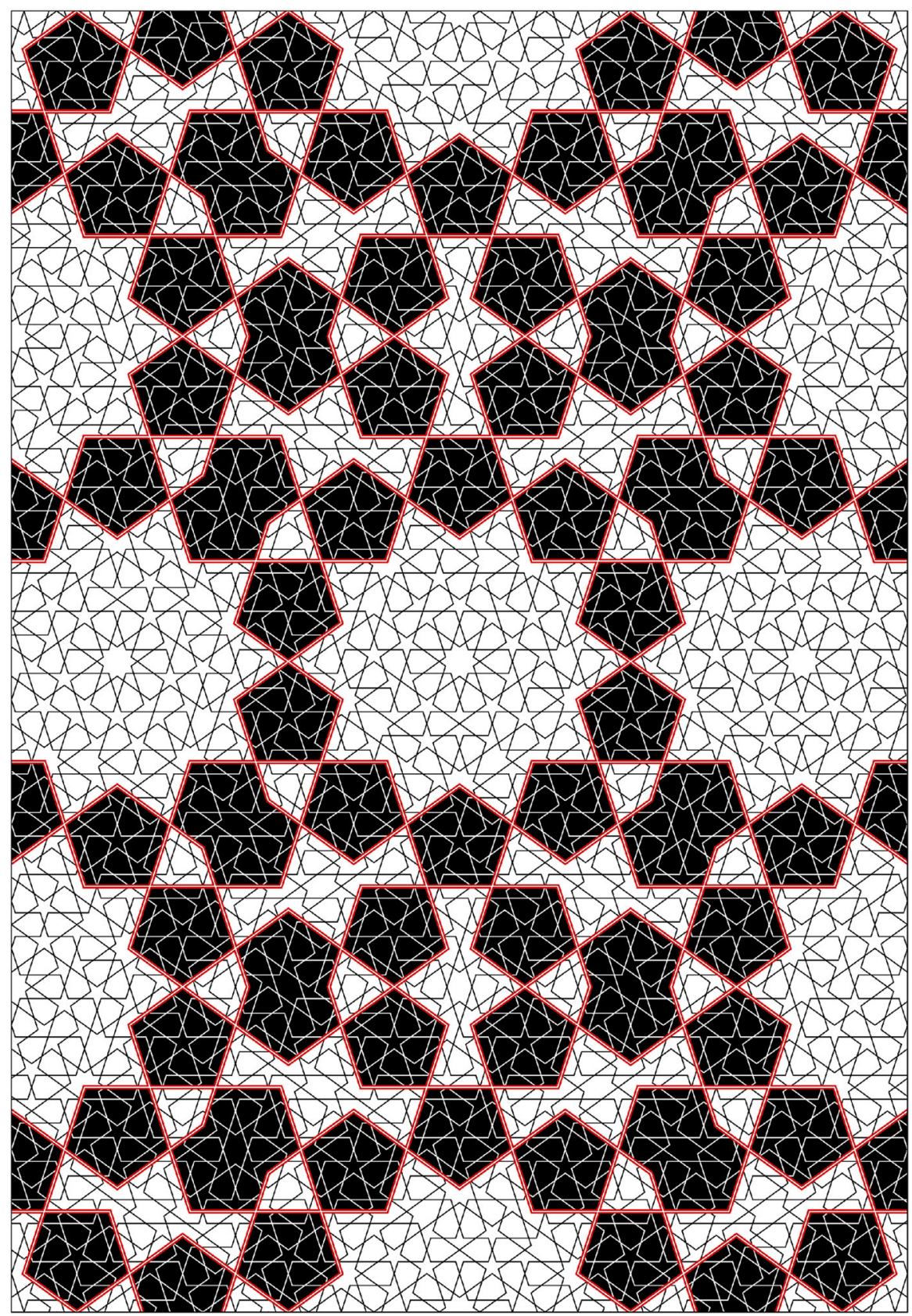

Fig. 31 After drawing the X-Lines on the rhombs of Fig. 25 we got a pattern of the family $\{F\}$ at the second level 


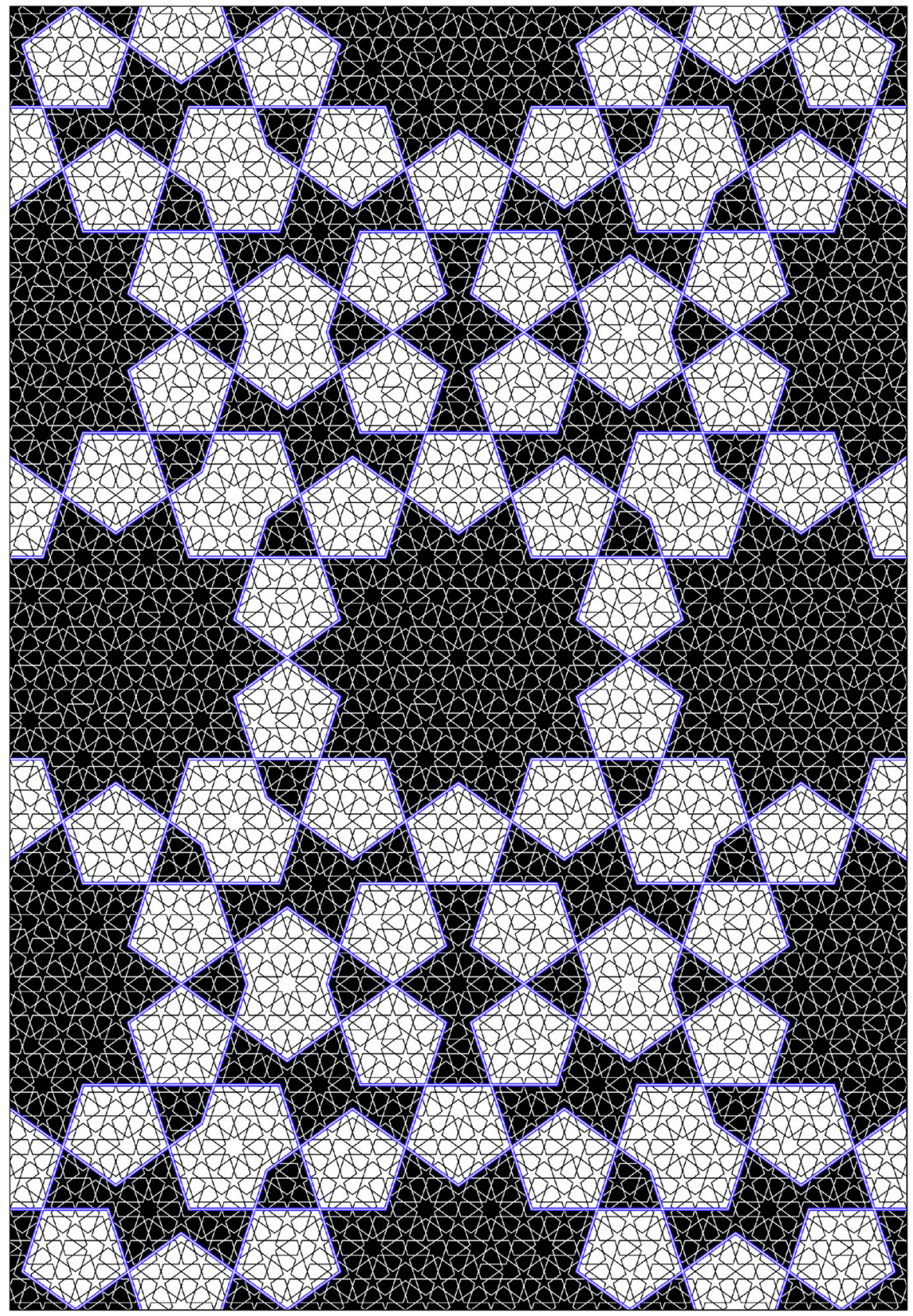

Fig. 32 Same transformation applied on Fig. 26. The second level pattern belong to the Iranian style "Tond" 


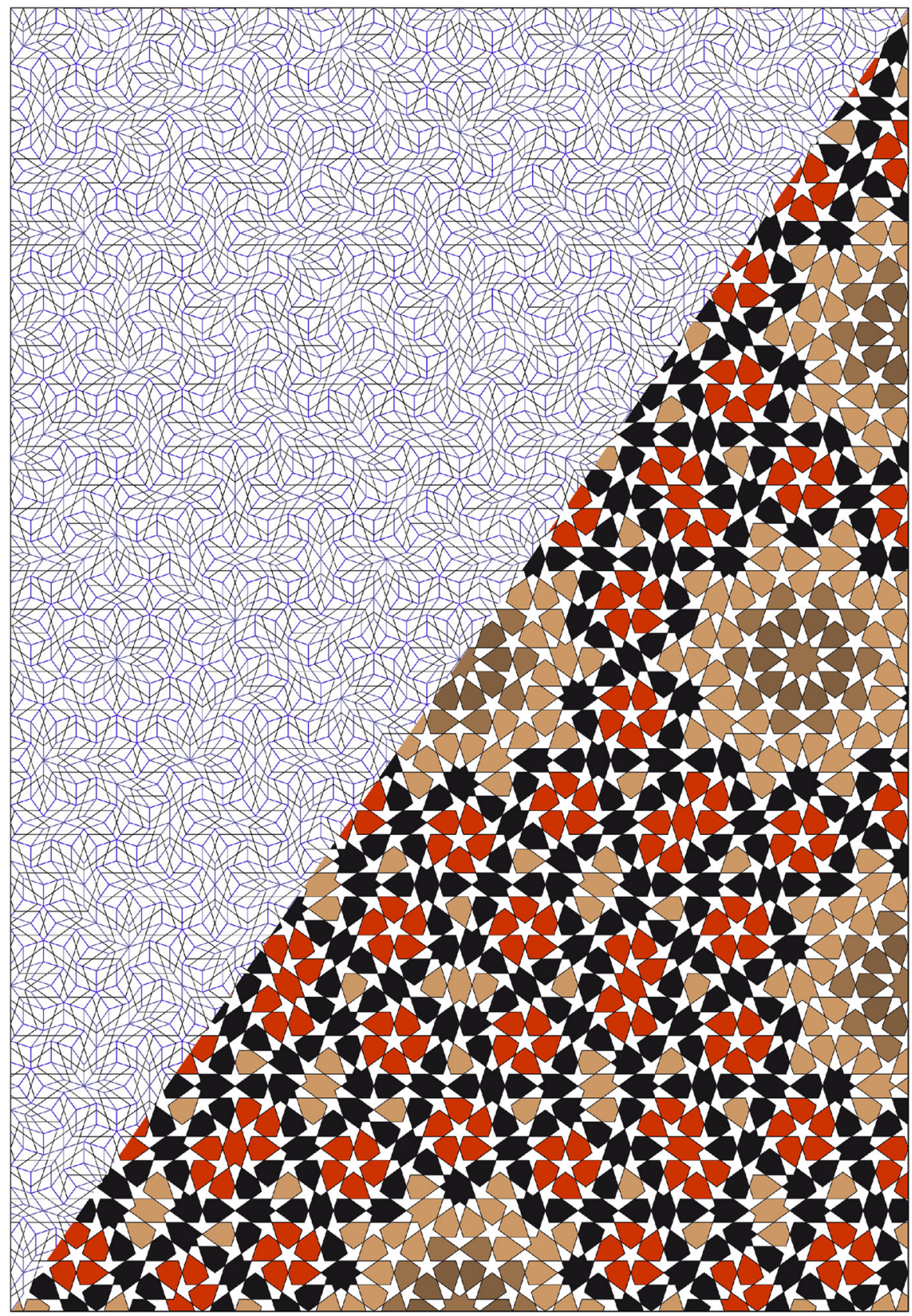

Fig. 33 Second level pattern of the Fig. 31. We have colored in black all the Positive Tiles crossing the edges of the first level 


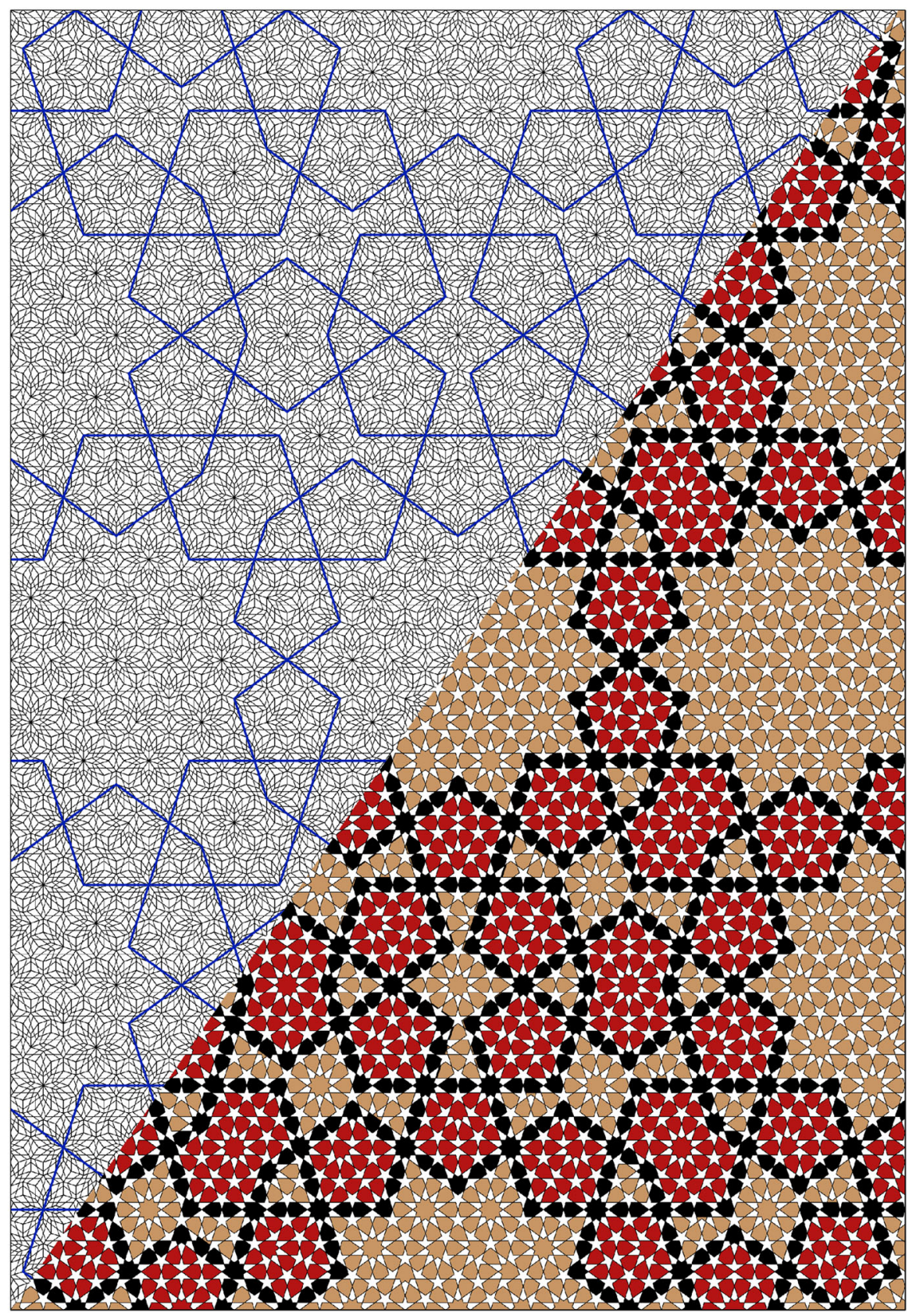

Fig. 34 Same style of coloration applied to the Fig. 32 


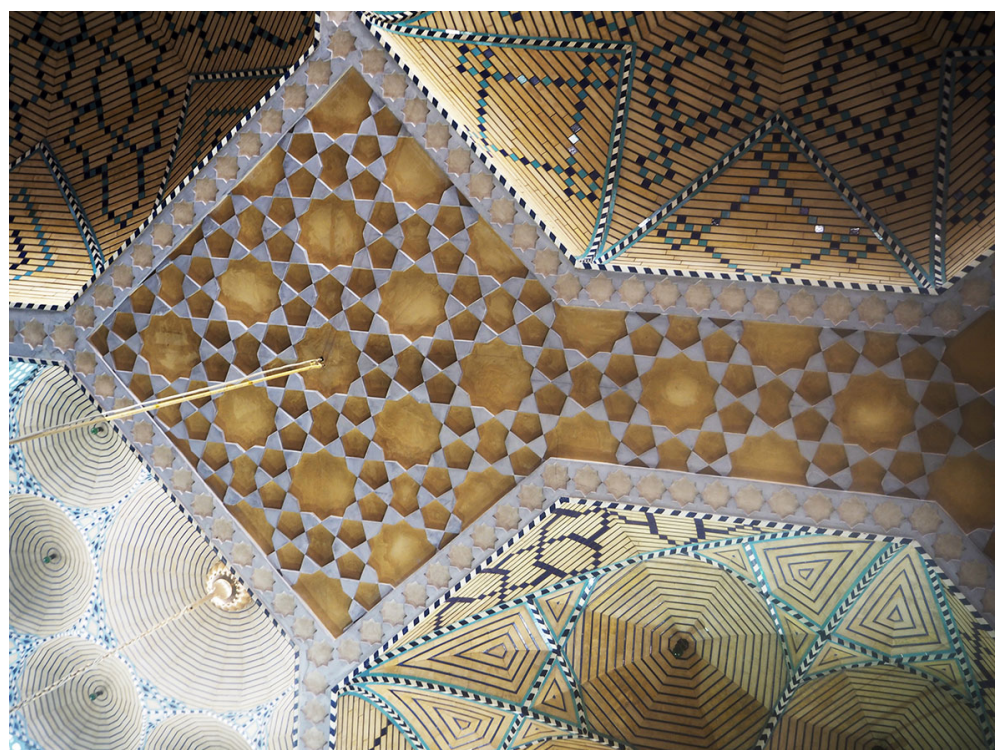

Fig. 35 "The mother of tillings" can be considered here as made from only one kind of shape, the "Toranj" (N2). Part of the roof of a new mosque in Isfahan

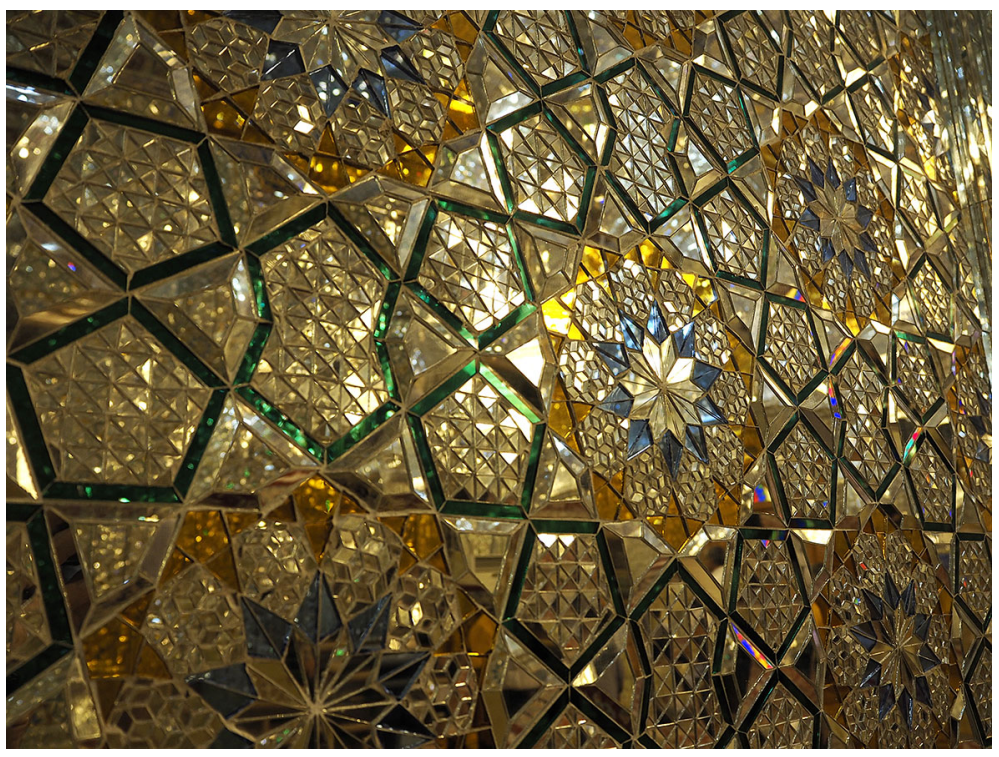

Fig. 36 The same pattern made of mirrors in Shiraz, Shah Cheragh shrine. With insertion of a rosette into the central star 


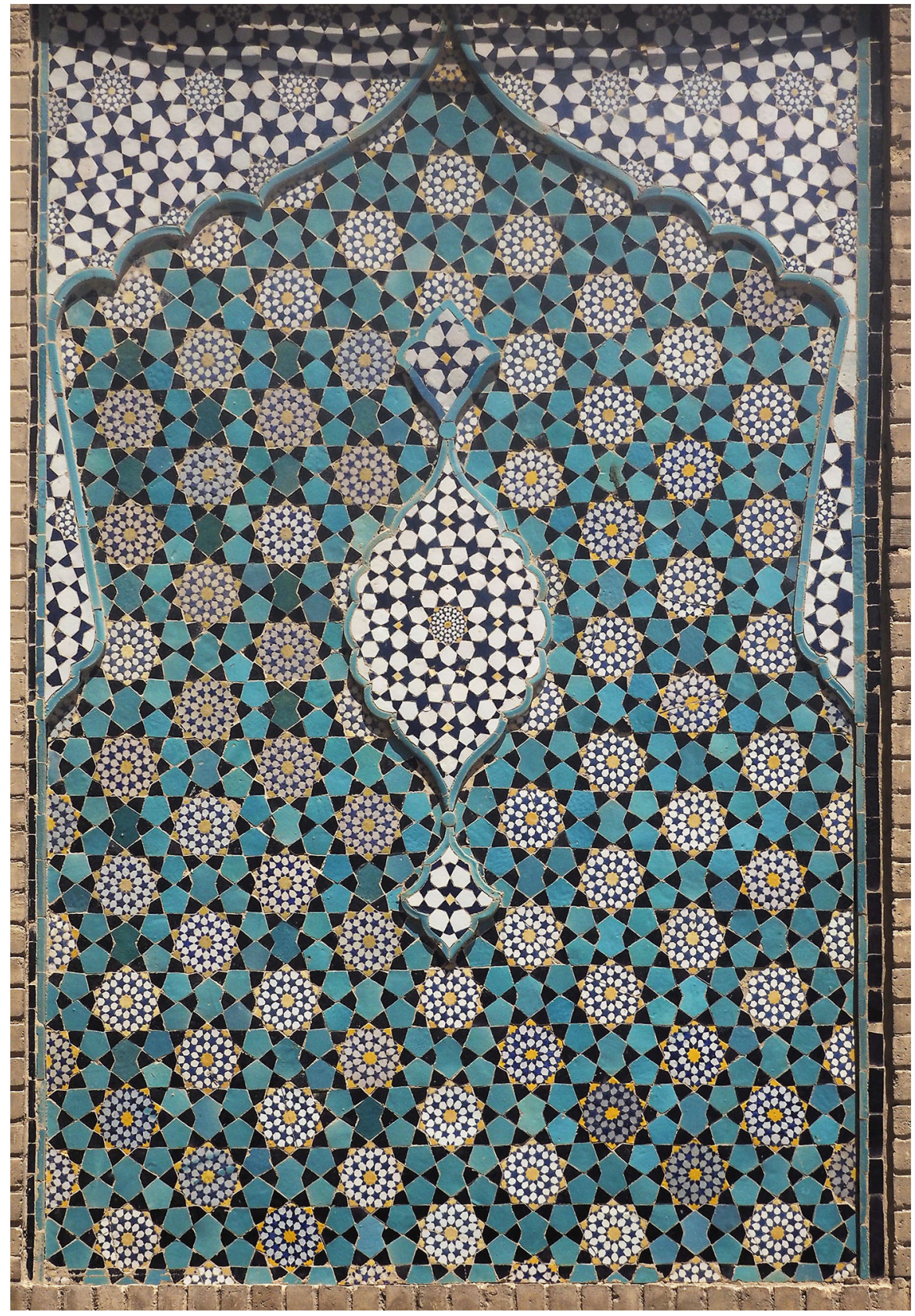

Fig. 37 Isfahan, Friday mosque. Same simplest Kond pattern, K1, with variation (type 1) of the Shamseh. The pattern at the background, the central medallion and the decoration of the Shamseh belong to the Kond and Shol Iranian family. According to this paper, every tile belong to the set [S] (with variations of $\mathrm{N} 1$ and N6), at different scales 


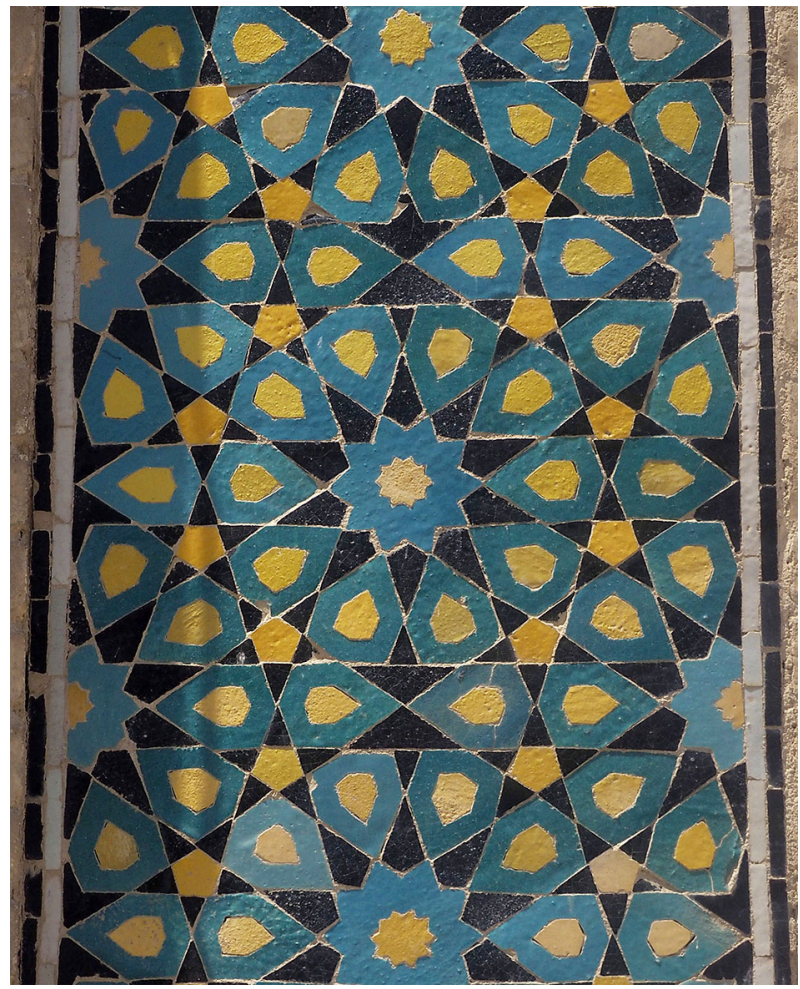

Fig. 38 Isfahan, Friday mosque

A same pattern may look very different according to the material and the techniques used, this constitutes also a kind of variation. The simplest Tond pattern can be seen made of mosaic of ceramic (Fig. 38), simply engraved on a wall (Fig. 39), in wood (Figs. 40, 41), in mirrors (Fig. 42), sometime with slight variations (Fig. 40). It can be decorated in a way that makes a link with the Kond family (Fig. 43), as explained below.

The rhomb L1 on Fig. 44 is the unit cell of the standard pattern T1 with each Tond tile decorated according to the rules shown at the bottom of the figure. The rhomb L2 is the complementary shape (see Castera et al. 2011). The mapping of each edge of the rhombs is identical and symmetric, so this decoration of the two rhombs can be applied to any Binary tiling and any Penrose tiling as well.

The tiling on Fig. 43 is not a perfect implementation of this process, because the decoration of some tiles uses another scale.

Note: This process does not works automatically for any Tond or Kond pattern.

\section{Strange Patterns}

The patterns are not only used to fill rectangular surfaces. Sometimes they have to adapt to a curvilinear shape, as for the medallion in Fig. 45. This Kond pattern is more 


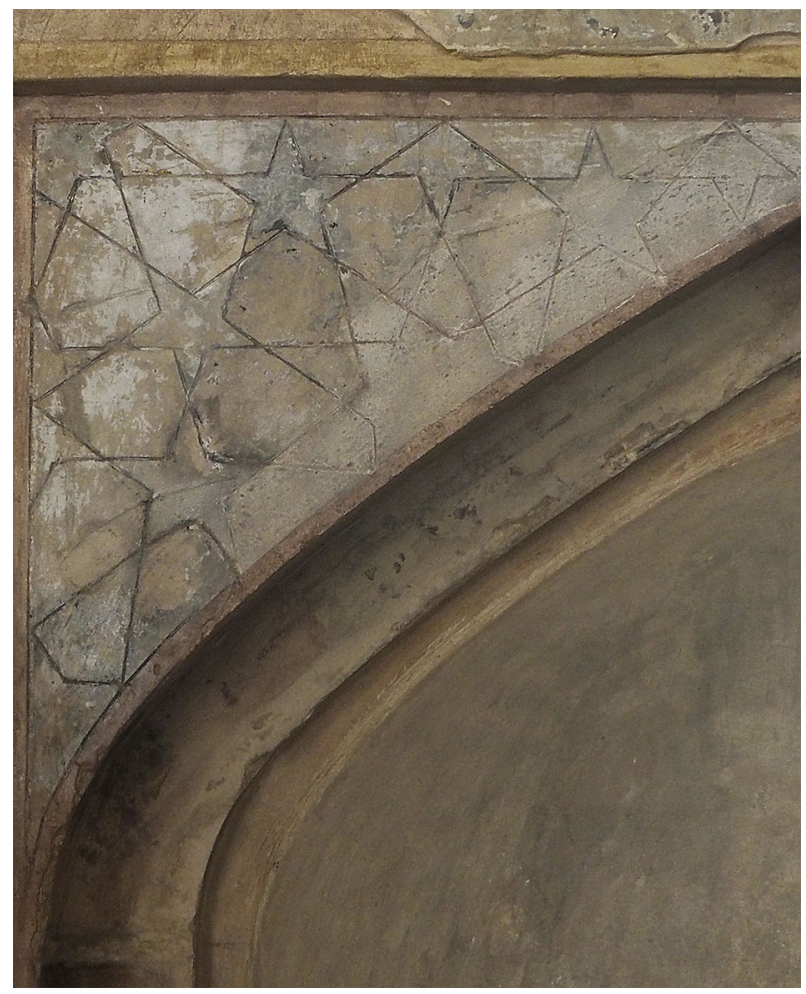

Fig. 39 Isfahan, Hasht Behesht (The eight paradises)

complex than the simple "mother of tilings" K1 which we have seen before. At first sight it seems to be made of the repetition of a rectangle with a Shamseh at each corner (Fig. 46a, 4 of such rectangles). But after simplification by removing all variations of N1 (Fig. 46b, c, d), it appears to be basically nothing but the simple K1 pattern with variations $\mathrm{N}_{1}$ and $\mathrm{N1}_{2}$ (Fig. 46d, the pattern is shown as repetition of a lozenge).

However, compared to K1 some areas are slightly different (Fig. 46e), and the "hidden" tiles N1 are decorated in a way that does not seems to follow an obvious logical rule (Fig. 46f).

We have chosen to highlight the orientation of the $\mathrm{N}_{2}$ variation with an arrow (Fig. 47).

I certainly do not consider this lack of symmetry as an error. In my opinion, this adds life to a pattern which is inserted into a vegetal shape.

In the same style, the pattern on Fig. 48, at the Friday mosque in Isfahan, is particularly remarkable. Again, at a first sight it can be seen as repetition of a rectangle (Fig. 49a). At the next step of simplification we can recognize the regular repetition of two overlapping stars (Fig. 49b). But the decoration of these shapes is not regular. In Fig. 49c we have used the same convention as before (Fig. 47) to show the orientations. Same thing in between, the arrangement of the tiles is not regular as shown with the black arrows (Fig. 49d). 


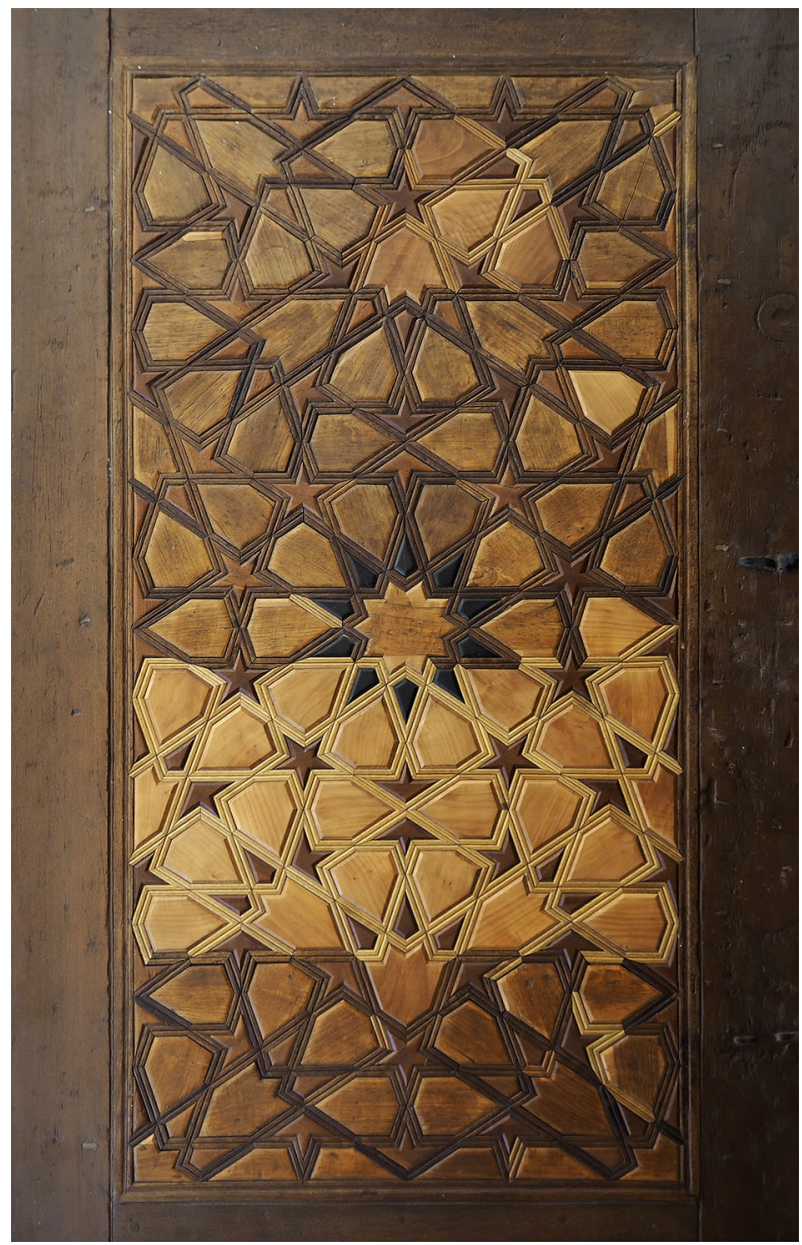

Fig. 40 A door in the old bazar of Isfahan

Again, there is certainly no error in this non-periodic tilling. This gives the viewer a strange feeling made of the contrast between the strict order of the main stars and the disorder of other tiles, sparkling like reflections on the water, or leaves in the wind. Could it be a coded message? Why not, I guess Muslims had the sense of humour in the old times. But I'm not the one who will even try to decode it.

This tiling would be difficult to analyze without using any simplification.

An other strange pattern can be seen on the spandrel of the main entrance of the Friday mosque in Isfahan.

All tiles belongs to the set [S1]. In this case, I cannot find any efficient simplification or other process that could reveal a hidden regular structure (Fig. 50).

Maybe a reader of this paper can make it. In my opinion, the artist started with the idea of a Kond pattern in which he could highlight a cursive drawing by the use of color. This cursive drawing could be considered the first level pattern. 


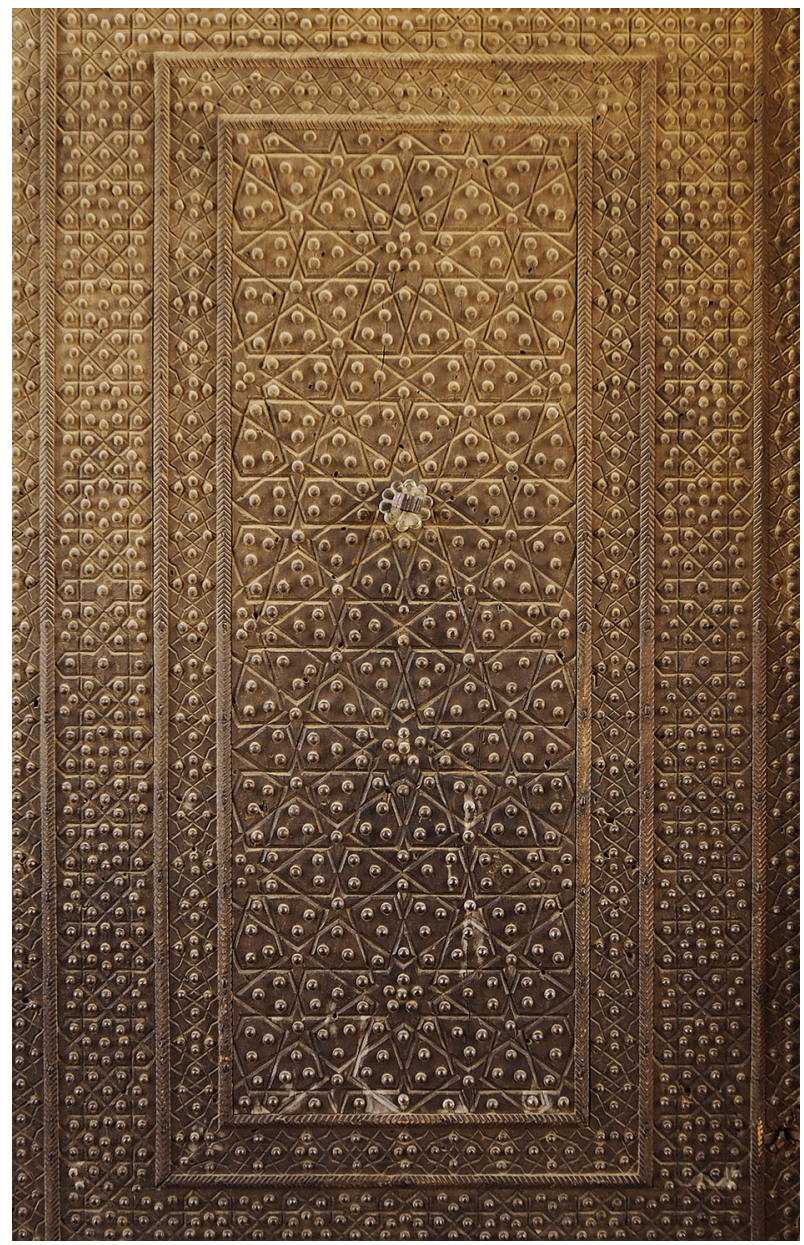

Fig. 41 Kachan, a door at Agha Bozorg shrine

\section{The X-Tiles in Historic Patterns}

The two wooden doors in (Fig. 51), although they are not from Iran but from the Serefeli mosque, Edirne, Turkey, belongs to the Persian style. The first pattern (Fig. 51a) is the simple T1, while the second (Fig. 51d) is a variation. On the images at the middle we have superimposed the Penta-Rhombs, so you can see the X-lines of the pattern inside. On the left, the reconstruction of the pattern with the tiles of the "X-Puzzle", a game I have specially made.

I do not pretend that the historic artists had used this process.

I wonder if it worth it to search a Penrose pattern in a tiling which can be reduced to a Binary tiling... 


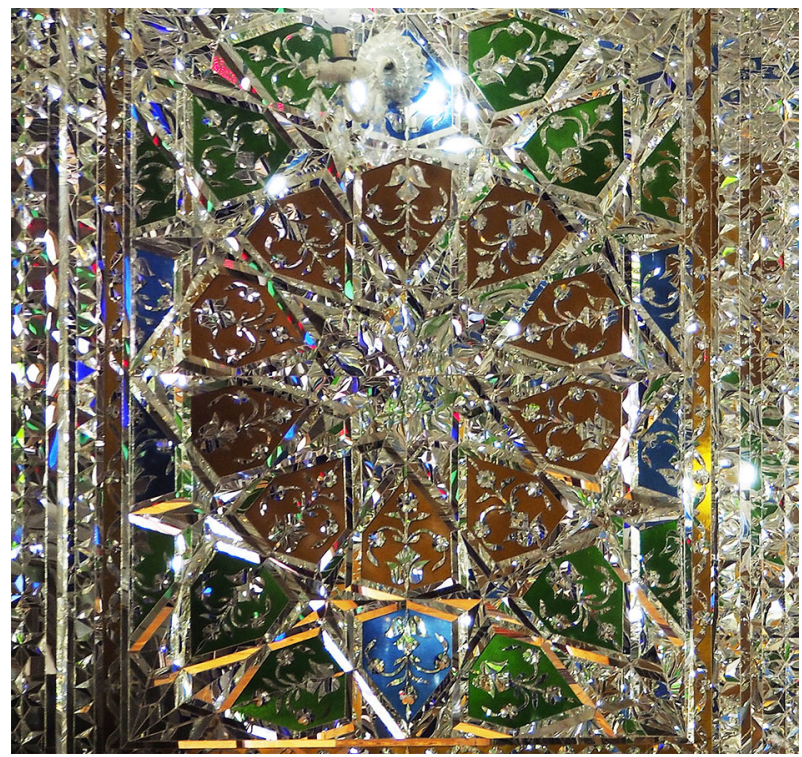

Fig. 42 Yazd, mirrors in a shrine

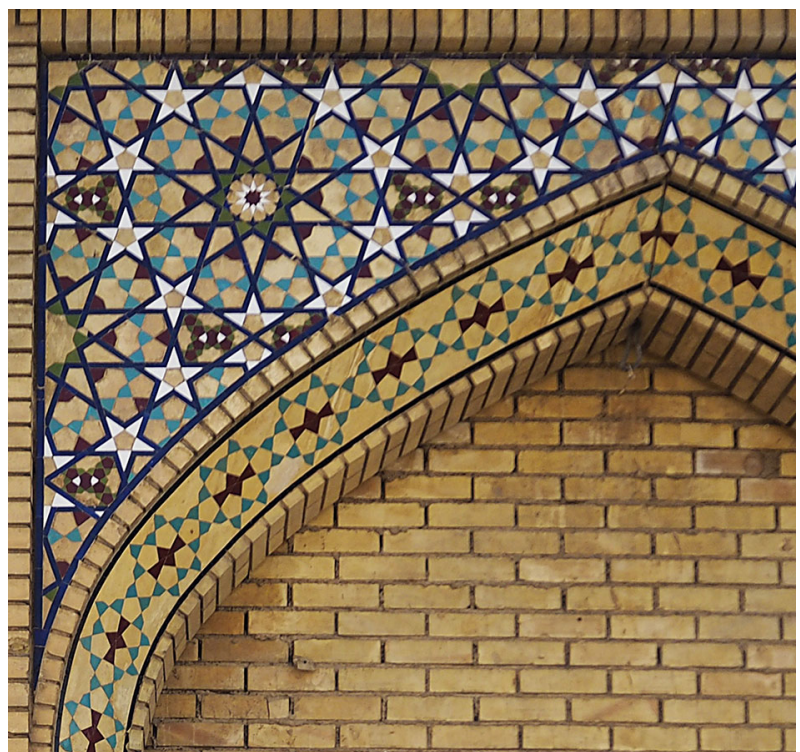

Fig. 43 Shiraz, in the old city

This pattern on a spandrel in Darb-e Imam, Isfahan (Fig. 52), is made of two level. The first level is a Kond tiling, again the simplest $\mathrm{K} 1$, while the second belongs to the Tond family. The filling of the central star has been adapted to the 


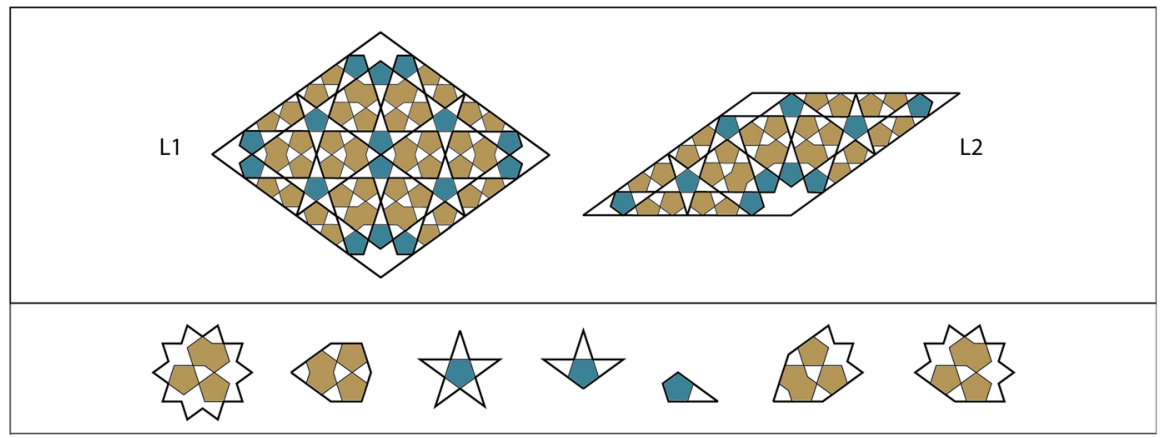

Fig. 44 A process of transition between the Tond (bold lines) and Kond (thin lines) patterns

shape of the spandrel. A quick sight at the tiles is enough to understand that this pattern could be made of our X-Tiles: indeed, they all belong to the set [X] (Fig. 29).

Figure 53a shows the basic pattern K1. In the Fig. 53b, c we have drawn the Penta-Rhombs, from which the pattern can be reconstructed when we add the $\mathrm{X}$-Lines to each rhomb (Fig. 52d). Notice that we have filled the Shamseh in a regular way, contrary to what we see in the actual tiling.

On the Fig. 53e we have removed the rhombs and added arrows to show the irregular orientation of the pentagons.

Figure 52f shows the whole pattern in a periodic version, inserted into a rectangle, with regular orientation of the pentagons and Shamseh. This pattern constitute a slight variation to the solution given in "Tiles Decomposition into "Penta-Rhombs"" (Figs. 23, 28, 32): the mapping of the tiles P2 and N2 are different.

In term of self-similar pattern, the characteristics of this one are:

Styles: From Kond to Tond (First level Kond style, second level Tond style)

1st level: K1 pattern, periodic. Tiles: P1, P2, N1 $1, \mathrm{~N} 2$

2nd level: All tiles belongs to [X].

This is a 2-level pattern, non self-similar because the tiles of the two levels do not belong to the same set.

We are going to see more multilevel patterns in the next section.

\section{Multilevel Patterns}

These examples are sometime call self-similar (Bonner 2003), but they are not in the strict sense defined in "Two Self Similarity Systems on $\{\mathrm{S}\}$ ". They are only 2-level patterns, although some of them could be easily transformed to be self-similar.

Convention: We call "System 1" the first system of Self-Similarity defined in "Two Self Similarity Systems on $\{\mathrm{S}\}$ " (Fig. 15), and "System 2" the second (Fig. 17). 


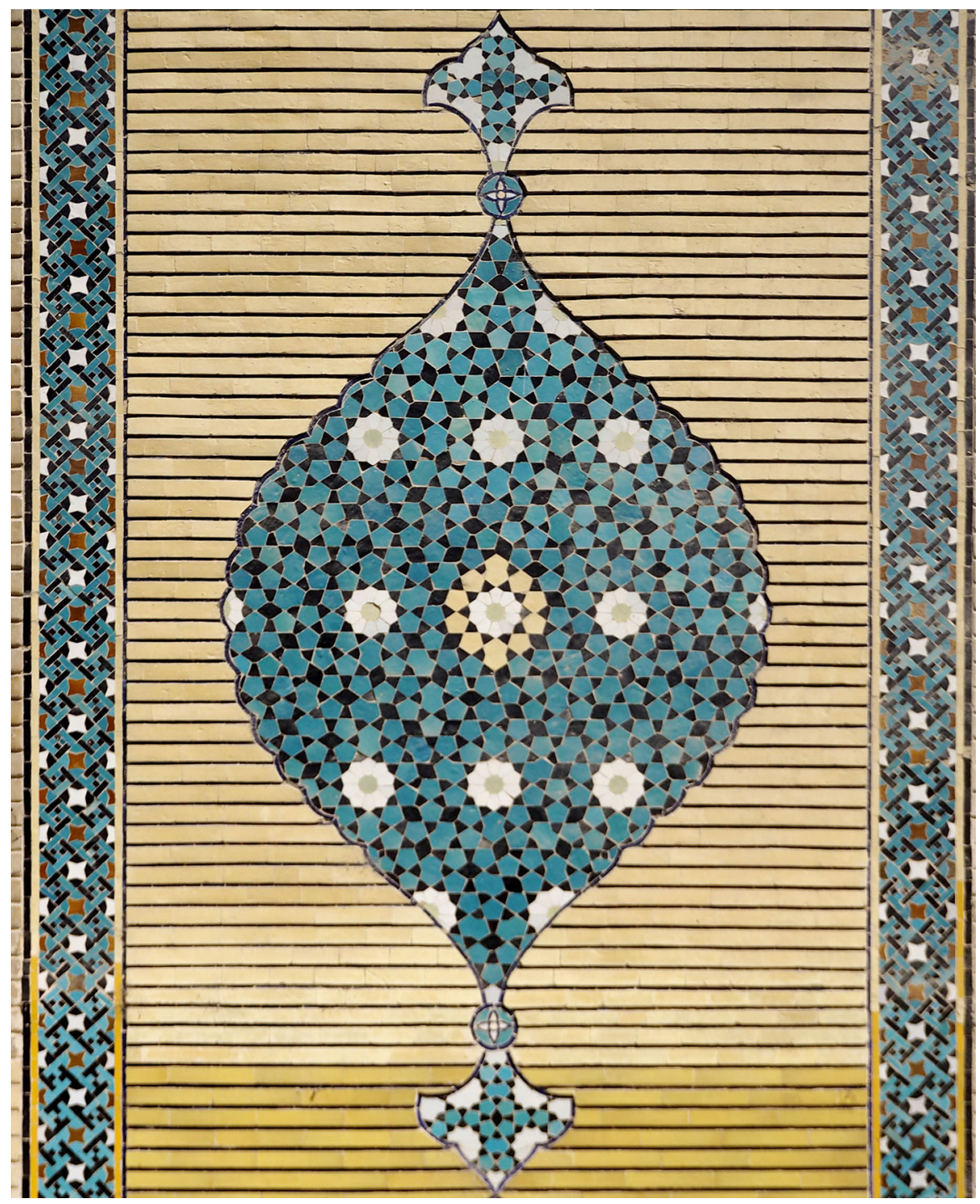

Fig. 45 A medallon in mosaic of ceramic at Chahar Bagh Madrasa, Isfahan

Example 1, Fig. 54. A spandrel at Shah Cheragh, Shiraz.

Characteristics:

Styles: From Kond to Kond.

Inflation rules: System 1.

1st level: K1 pattern, periodic. Tiles: $\mathrm{P} 1, \mathrm{P} 2, \mathrm{~N} 1_{1}, \mathrm{~N} 2$

2nd level: All [S1] tiles: P1, P2, N1 1, N2, N3.

Second option of inflation for the tile P1 (P1b on Fig. 15). 


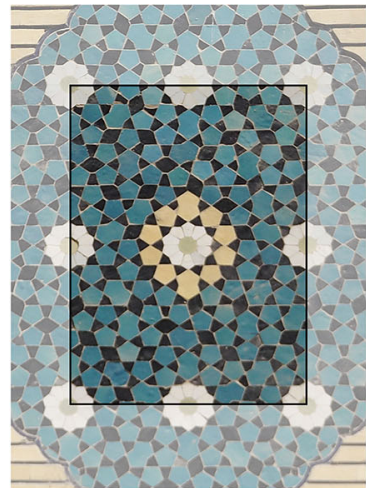

a

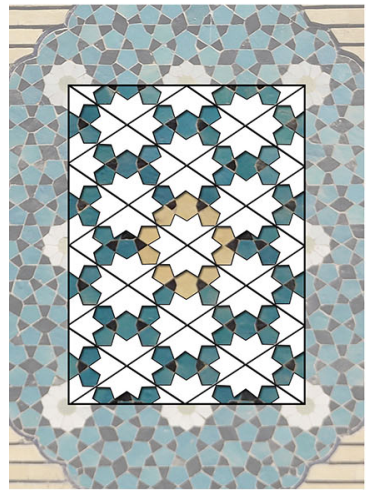

d

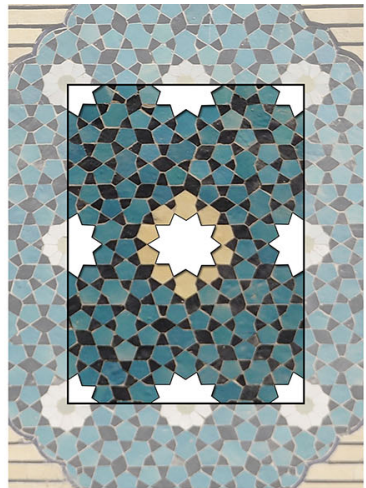

b

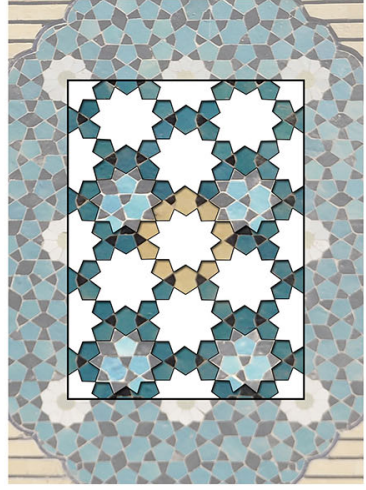

e

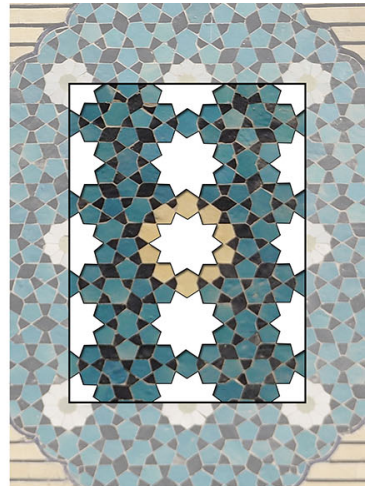

c

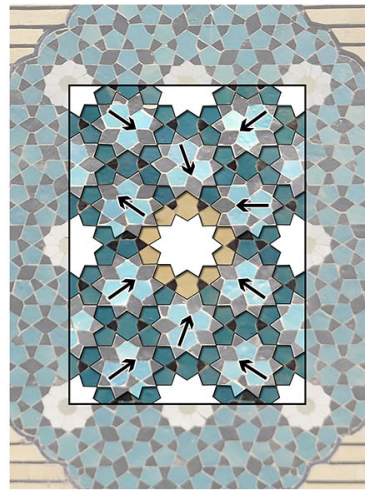

f

Fig. 46 The pattern of the medaillon, considered as a variation of the "mother of tilings"

Fig. 47 Orientation convention for the variation $\mathrm{N}_{2}$ used in 46-f

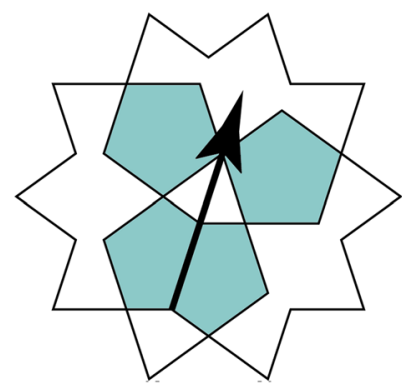

Two-level pattern, globally periodic. Not Self-Similar because the inflation rule is not define for the tile N3 (Sormedan), and the first level, periodic, cannot be mapped onto the second one. 


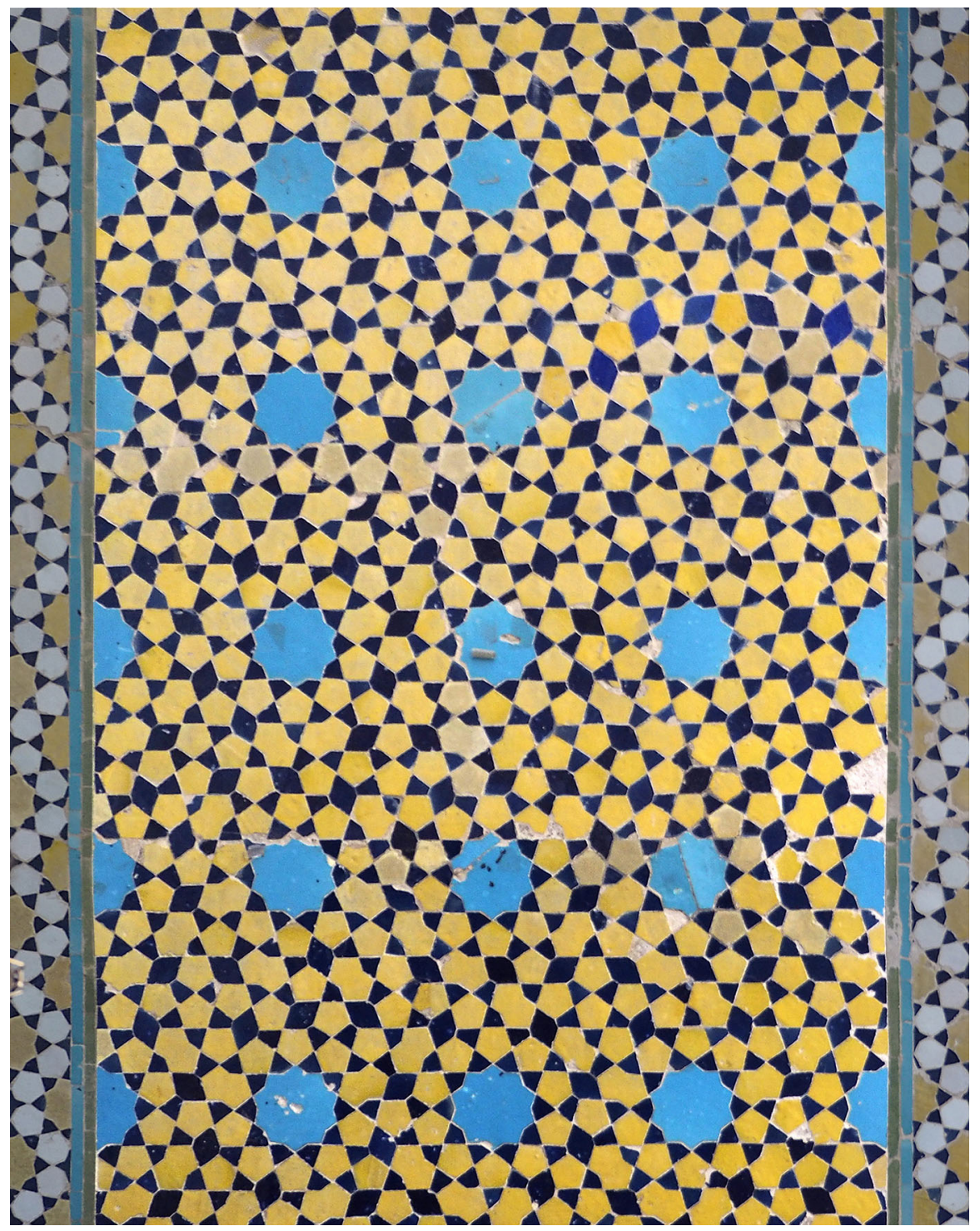

Fig. 48 Friday mosque, Isfahan

Particularities: The mapped pentagons P1b are laid out always along the vertical axis of the wall (Fig. 55b). This is the artist's choice.

Can we define a Self-similar pattern from it, with some modifications? Yes: System 1 with a slight variation for N1, and the use of the mapping P1b. 


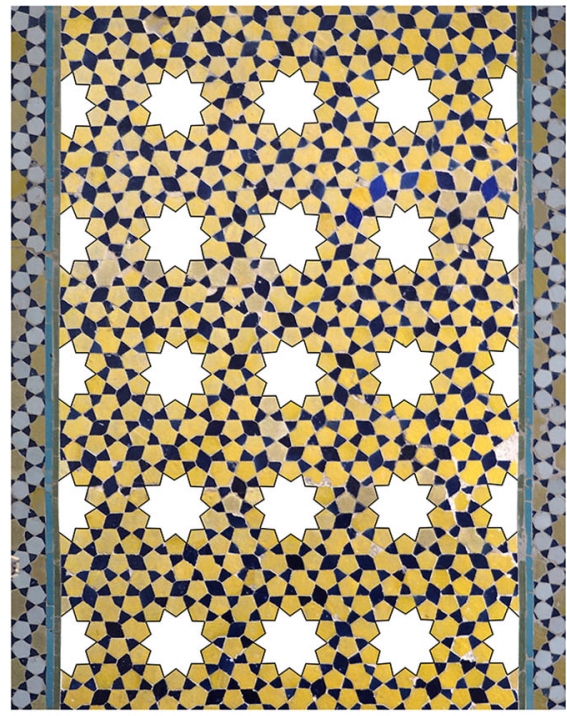

a

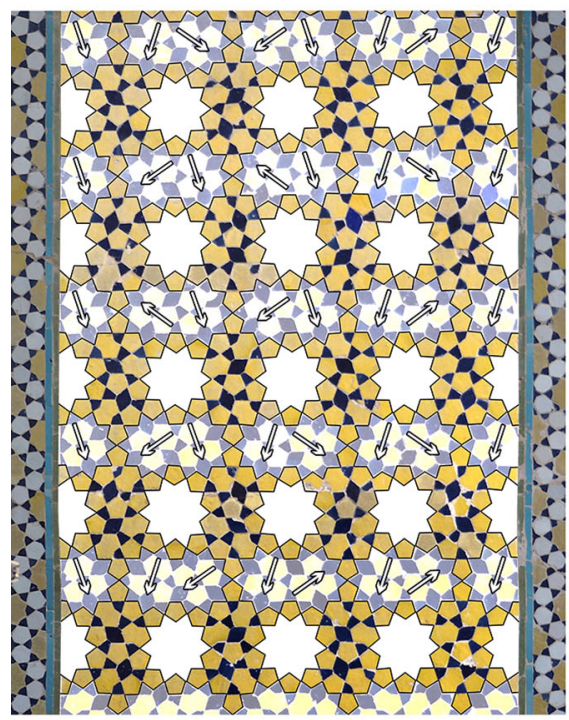

c

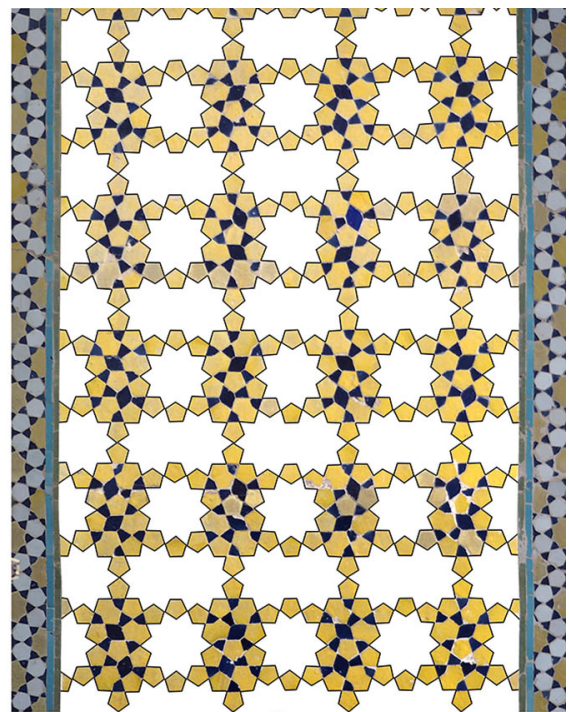

b

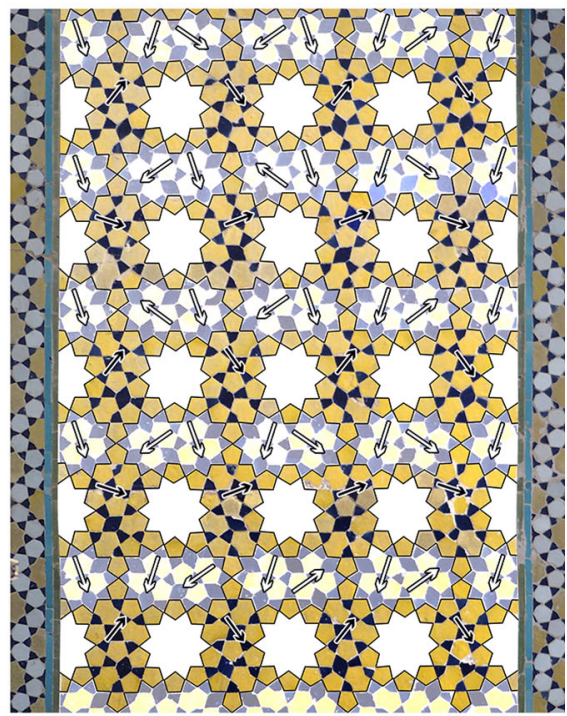

d

Fig. 49 a Periodic repetition of the stars. b Overlapping stars in between. $\mathbf{c}$ Non periodic orientation of the decoration of the overlapping stars and... $\mathbf{d}$ of the tiles in between

\section{Example 2, Fig. 56. Underside of an arch at the Vakil mosque in Shiraz.}

It is the same as the previous one with slight differences in how the mapped P1b are laid out.

We have drew arrows to show the orientations of the pentagons, and highlighted the variations $\mathrm{Pl}_{2}$ at the centre, to show the differences with the option on "Two Self Similarity Systems on $\{\mathrm{S}\}$ ”, Fig. 15. 


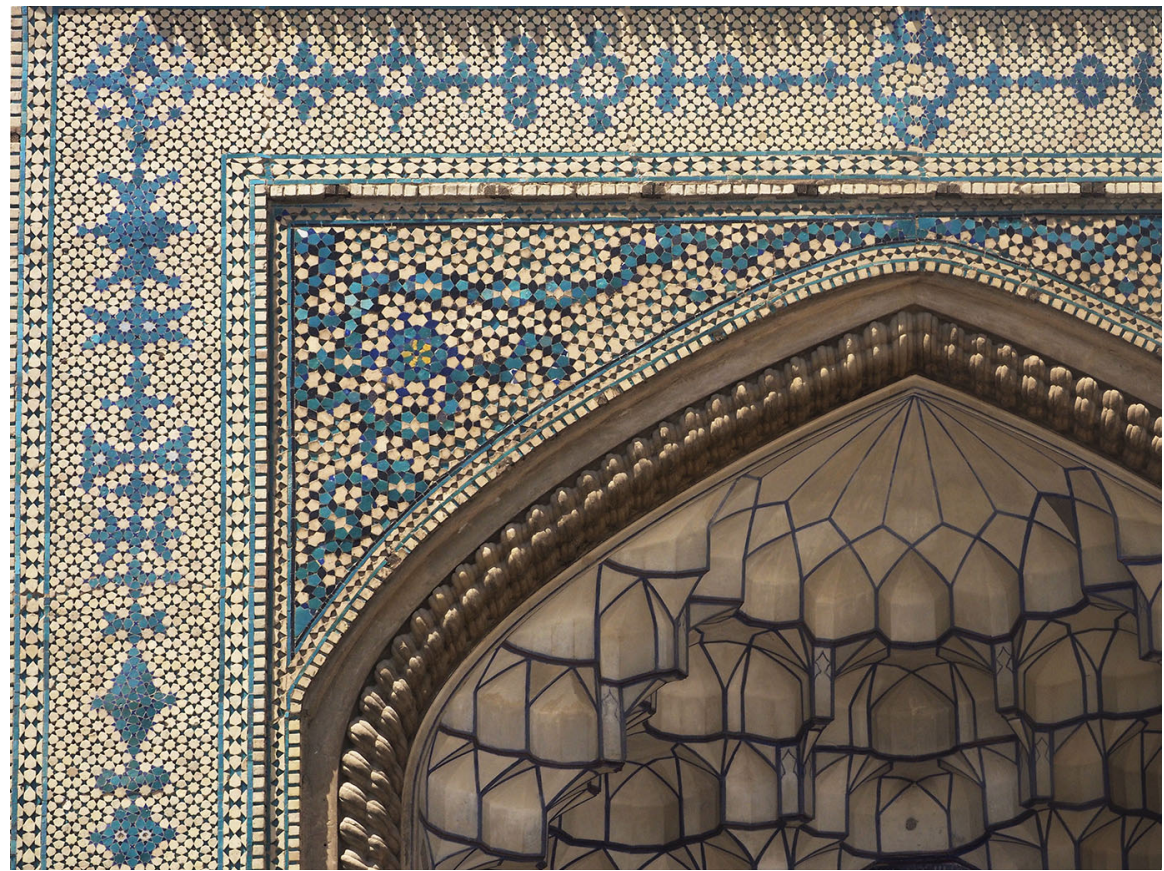

Fig. 50 Entrance Iwan of the Friday mosque, Isfahan

\section{Characteristics:}

Styles: From Kond to Kond.

Inflation rules: System 1.

1st level: K1 pattern, periodic. Tiles: $\mathrm{P} 1, \mathrm{P} 2, \mathrm{~N} 1_{1}, \mathrm{~N} 2$.

2nd level: All [S1] tiles: P1, P2, N1 $1, \mathrm{~N} 2, \mathrm{~N} 3$.

Second option of inflation for the tile P1 (P1b on Fig. 15).

Two-level pattern. Not Self-Similar because the inflation rule is not define for the tile N3 (Sormedan), and the first level, periodic, cannot be mapped onto the second one. Particularities: The mapped pentagons P1b are laid out in a puzzling way (Fig. 56, right), which makes this pattern non periodic.

Can we define a Self-similar pattern from it, with some modifications? Yes: System 1, with a slight variation for N1, and the use of the mapping P1b.

\section{Example 3, Fig. 57: the famous Darb-e Imam pattern in Isfahan.}

Characteristics:

Styles: From Kond to Kond.

1st level: K1 pattern, periodic. Tiles: $\mathrm{P} 1, \mathrm{P} 2, \mathrm{~N} 1_{1}, \mathrm{~N} 2$

2nd level: All [S1] tiles: P1, P2, N1 $1, \mathrm{~N} 2, \mathrm{~N} 3$.

Inflation rules: System 2, with an alternative option for the pattern of P1 (P1c in “Two Self Similarity Systems on $\{\mathrm{S}\}$ ", Fig. 17). 


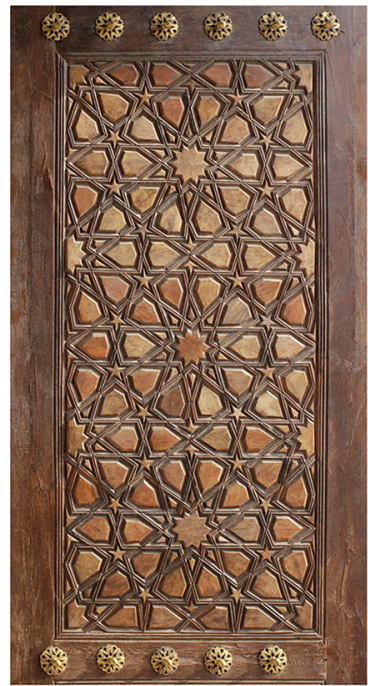

a

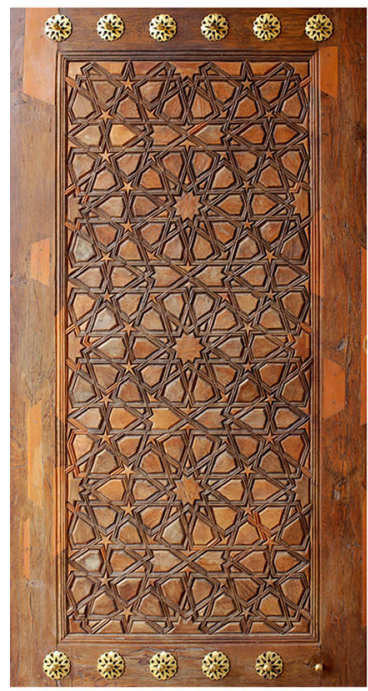

d

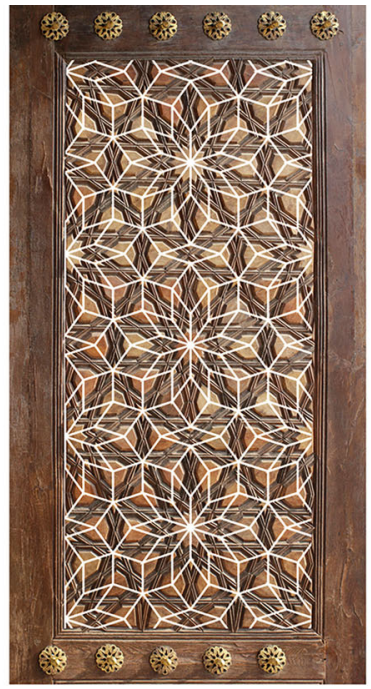

b

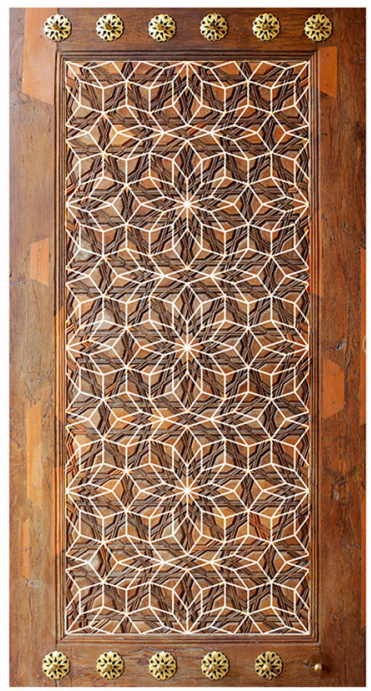

e

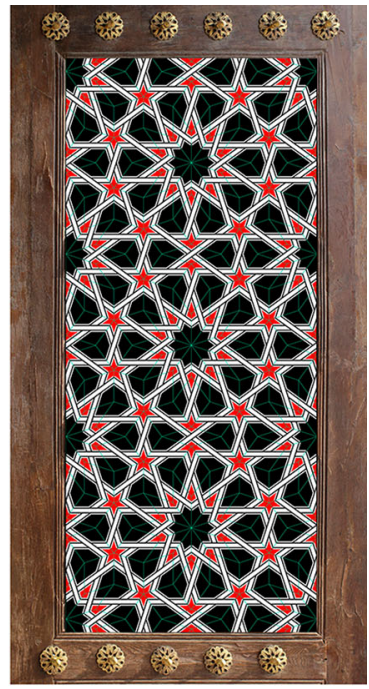

c

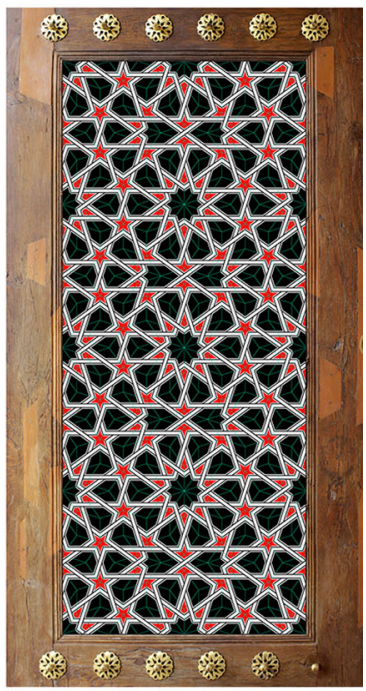

f

Fig. 51 Two doors at Serefeli mosque, Edirne, Turkey. Analysis and reconstruction in terms of X-tiles

2-level pattern, globally periodic. Not Self-Similar because the inflation rule is not define for the tile N3 (Sormedan), and the first level, periodic, cannot be mapped onto the second one. 


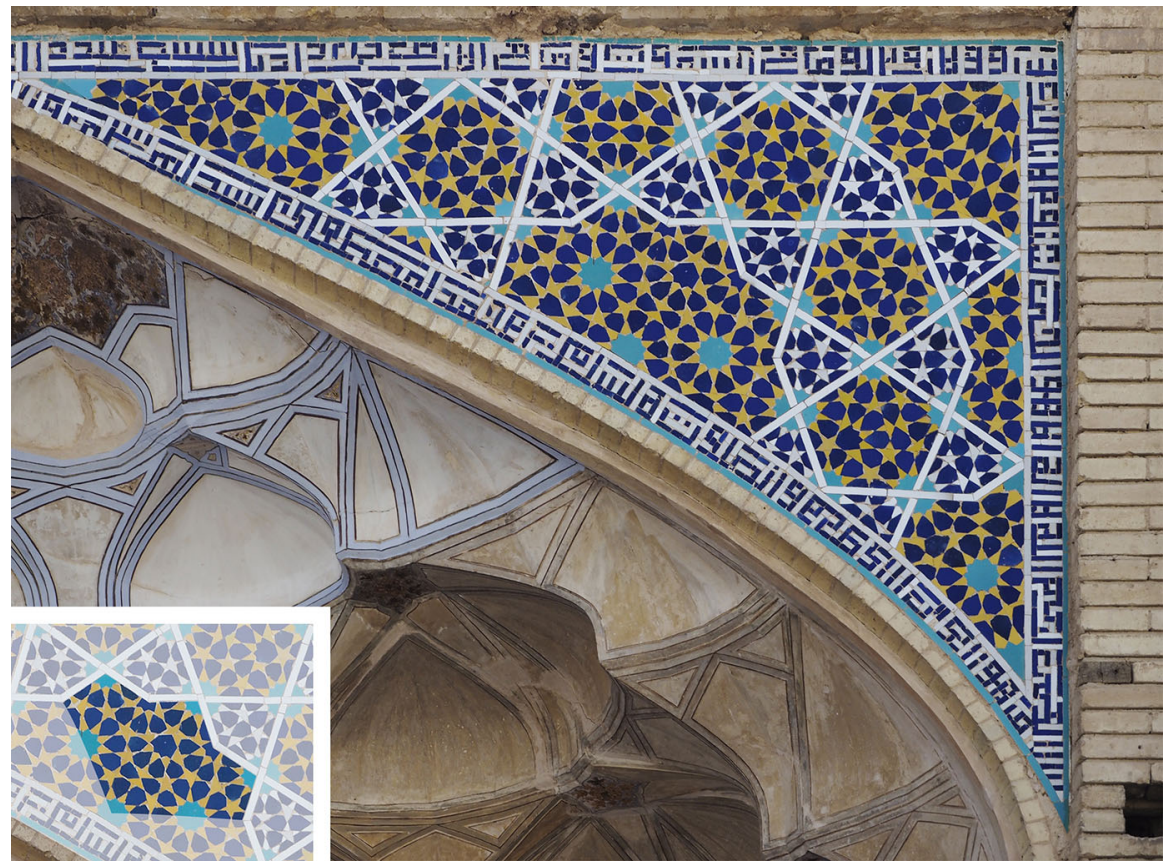

Fig. 52 A spandrel at Darb-e Imam, Isfahan

Particularities: The use of two mapping for the pentagons, P1c and its symmetric ( 1 and 2 on Fig. 57b). The orientations of those shapes (white arrows on Fig. 57b) is a little bit puzzling. In my opinion this cannot be an error from the artist, but a deliberate choice.

Can we define a Self-similar pattern from it, with some modifications? The problem is the use of two different mapping for the same pentagon. If we chose only one of them, the System 2 works easily.

Example 4, Fig. 58: A tiling on a wall in Chahar Bagh Madrasa, Isfahan.

We have highlighted in white the basis of the figure. The mapping of the edges is different to what is used in systems 1 or 2: The stars on the edges are connected with a tile N6 in between. In black, the special tiles used on the mapping of P2 (The same as for the variations $\mathrm{N}_{3}$ and $\mathrm{N6}_{1}$ ).

Characteristics:

Styles: From Kond to Kond + Shol (or from [S1] to [S]).

1st level: K1 pattern, again.

2nd level: All the tiles of [S] are present except P4 and P5. Variation $\mathrm{N}_{1}$, as usual, on both levels, variation $\mathrm{P}_{1}$, and an extra tile in the mapping of $\mathrm{P} 2$ (although there is a solution with no extra tiles). 


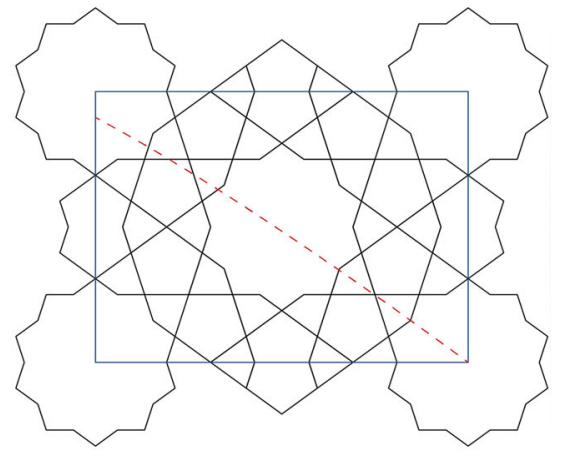

a

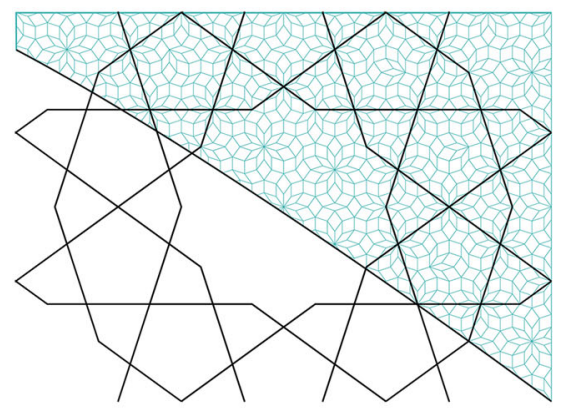

c

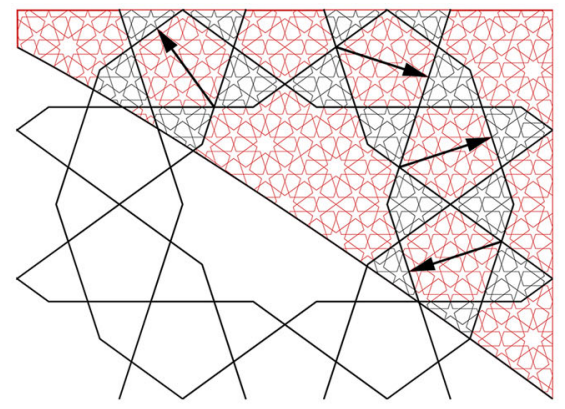

e

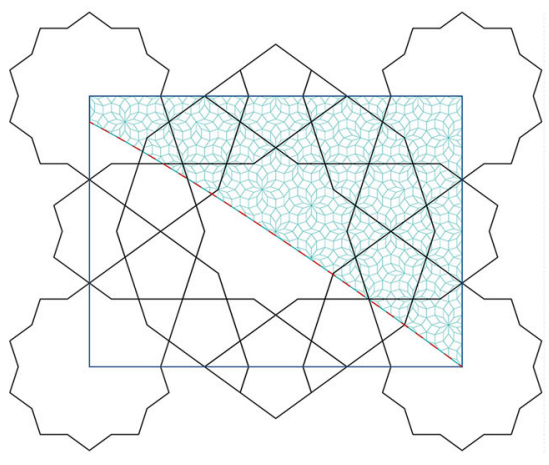

b

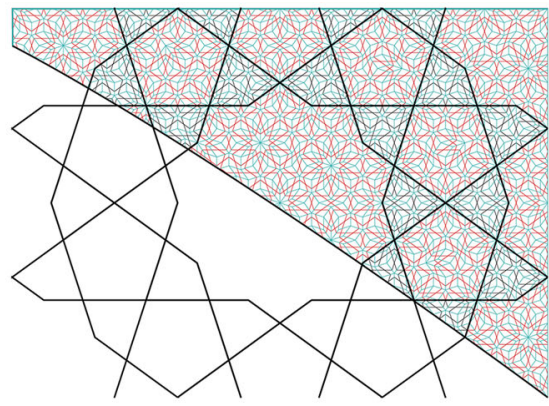

d

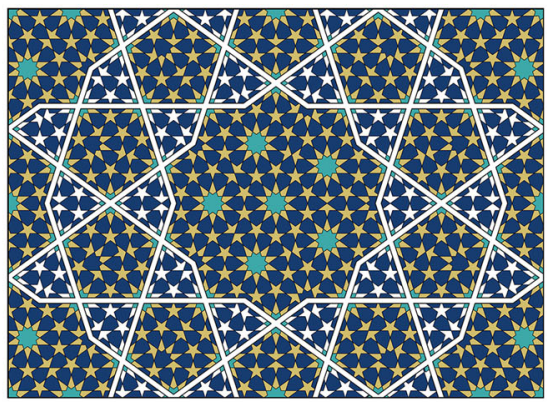

$\mathbf{f}$

Fig. 53 Analysis and reconstruction with the X-Tiles

Inflation rules: New system. The scale ratio is the same as for the system 2 .

Two-level pattern, globally periodic. Not self-similar because the inflation rules are not defined for most of the tiles at the second level (P3, N3, N4, N5, N6 and the extra tiles). Moreover, as usual the periodic first level cannot be mapped onto the second.

Can we define a self-similar pattern from it, with some modifications?

I will leave to the reader the pleasure to work on this question. 


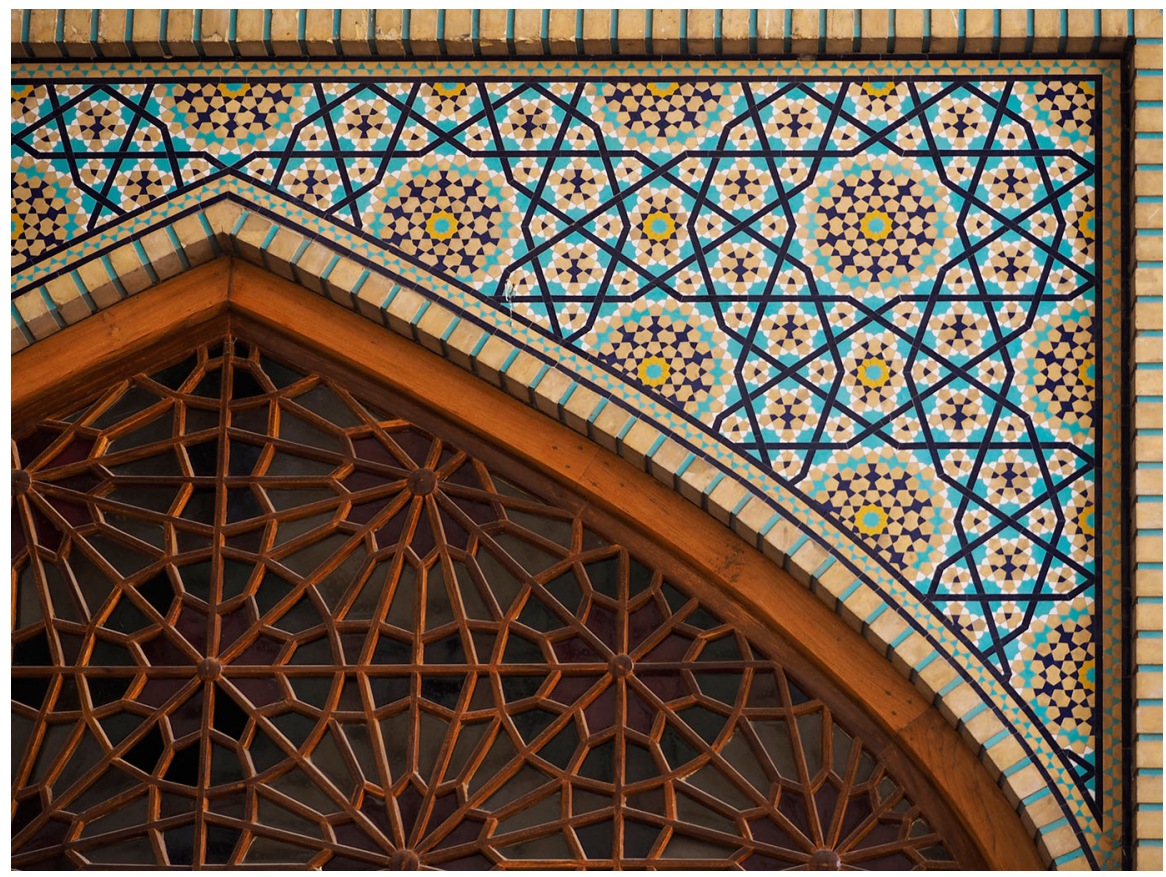

Fig. 54 Example 1: Shiraz, Shah Cheragh

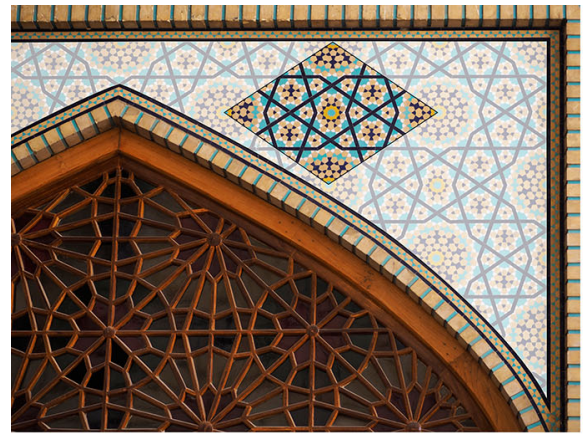

a

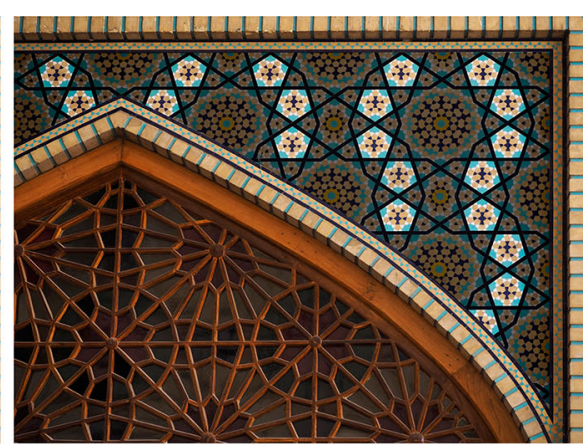

b

Fig. 55 a The unit cell of the "Mother of Tiles" K1. b Vertical orientation of the pentagons

At this point a question arise: Why artists haven't done a strict self-similar pattern? At least a pattern with a Sormedan at the first level, even though the whole pattern would be inserted into a periodic tiling?

I do not have an answer. Maybe there is such a pattern somewhere. 


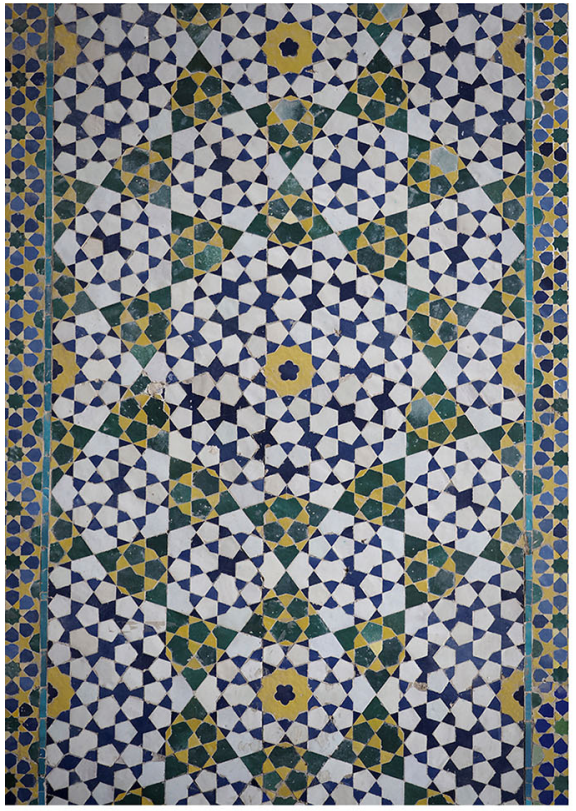

a

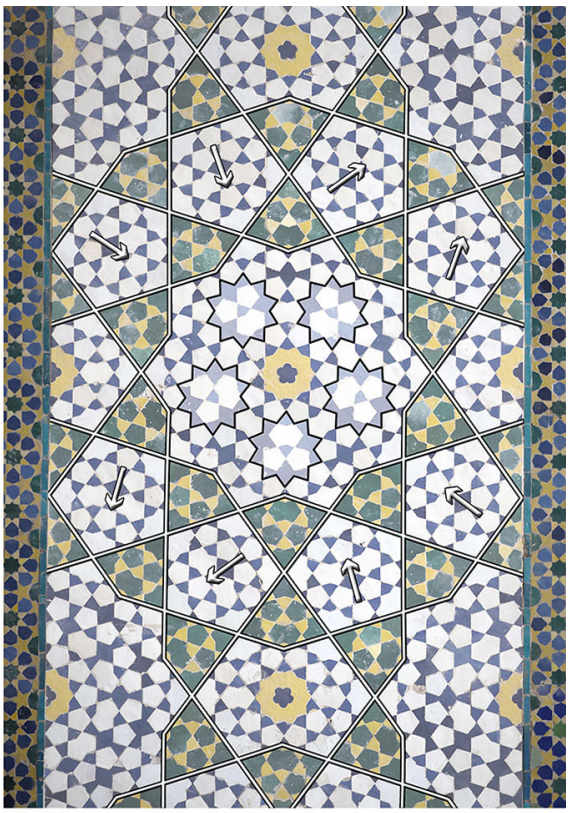

b

Fig. 56 Shiraz, Vakil Mosque, part of the underside of an arch. Right Orientation of the pentagons, and stars at the centre to be compared to Fig. 15, "Two Self Similarity Systems on $\{\mathrm{S}\}$ "

\section{Example 5, Fig. 59: Friday mosque, Isfahan.}

This is a rare example with a Sormedan at the first level (which contain only 2 tiles). The basis of the mapping is particular: the stars on the edges are oriented differently than usual, and are connected by a tile P2.

But the artists find difficulties in mapping the Sormedan. Their solution is quite tricky (in the skillful sense).

This can be compared to Bonner's "Self-Similar" (in a weak sense) Fig. 13, on his web site.

Characteristics:

Styles: From Kond to Kond.

1st level: Periodic pattern, tiles P1 and N3.

2nd level: Tiles P1, P2, $\mathrm{N}_{1}, \mathrm{~N} 2, \mathrm{~N} 3$ and exotics.

Inflation rules: New system, rule clearly defined only for the tile P1.

\section{Self-Similarity with Interlaces}

The photography (Fig. 60) shows a 2-level tiling with interlaces at the Madrasa Chahar Bagh, Isfahan. The two levels belong to the Kond family (tiles from the set [S1]) but the tiling is not self similar in the strict sense because of the two usual reasons: the inflation rule is missing for the tile N3 (Sormedan), and the first level is periodic. In the next figure we have highlighted the basic pattern K1 (simplified), the 


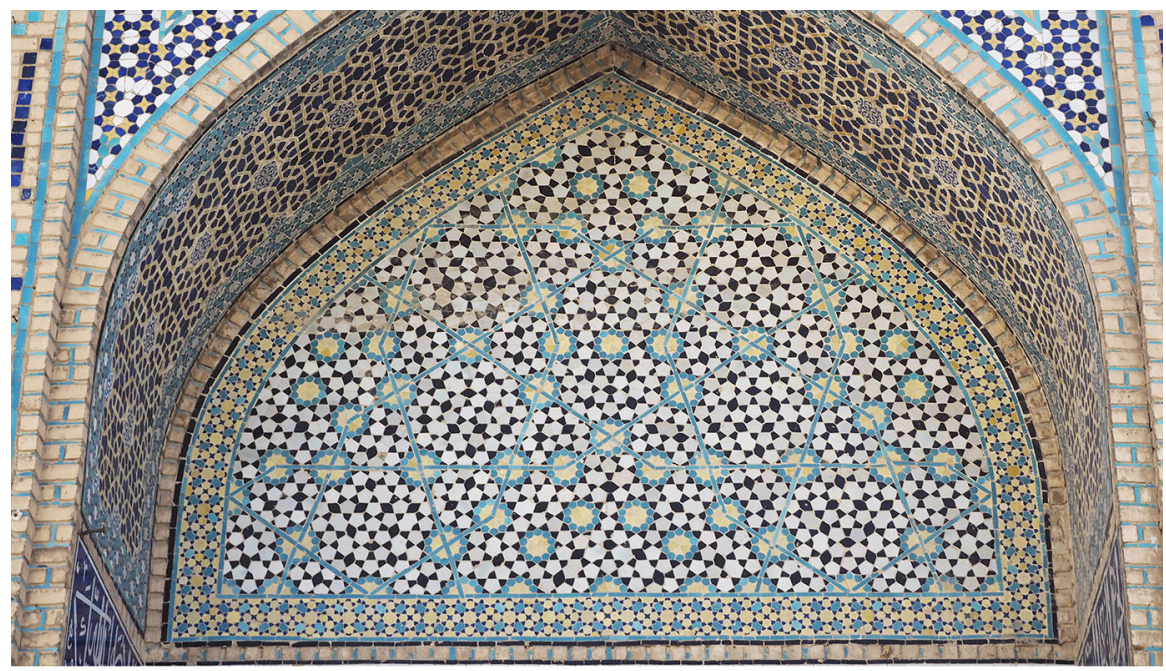

a

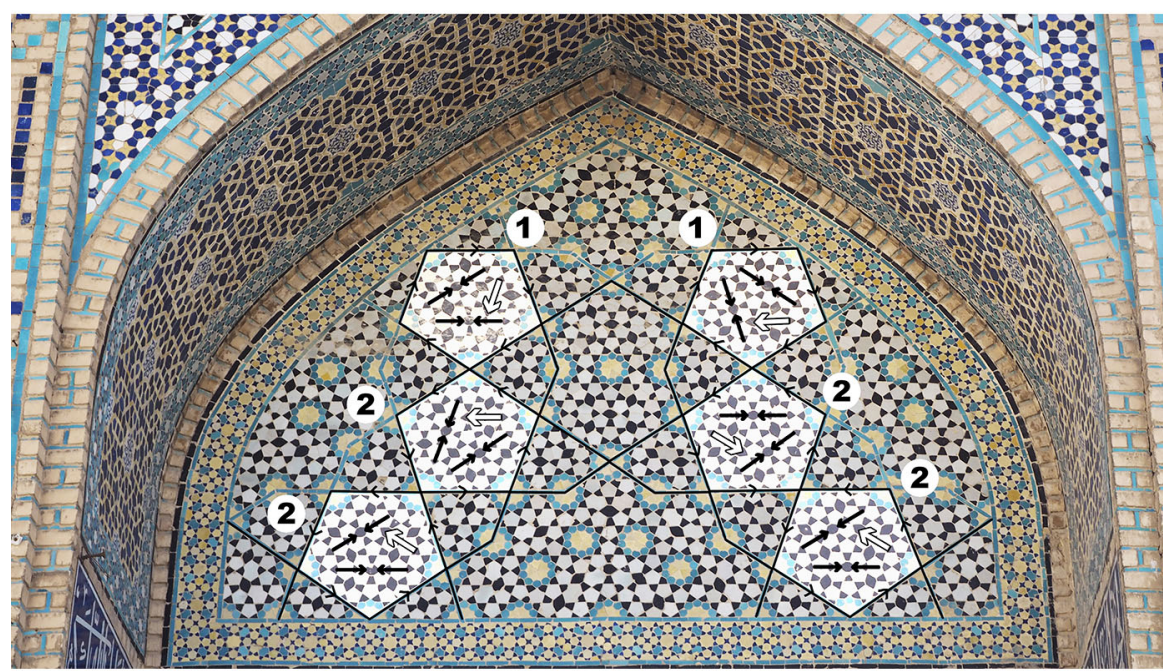

b

Fig. 57 Isfahan, Darb-e Imam

interlaces, and $\mathrm{p} 1, \mathrm{p} 2, \mathrm{n} 2 \ldots$ the inside shape of $\mathrm{P} 1, \mathrm{P} 2, \mathrm{~N} 2 \ldots$ after adding the interlaces.

Let's start the analyze by asking this question: can we find a self-similar Kond pattern with interlaces (Fig. 61)?

We can see on Fig. $62 \mathrm{~b}$ that the shapes $\mathrm{p} 2$ and $\mathrm{n} 3$ have not the same proportions than the original P2 and N3, although the angles are the same. The proportions of $\mathrm{p} 1$ and $\mathrm{n} 2$ are still the same as P1 and N2, but they are no longer at the same scale. The length of the corresponding edges are different. 


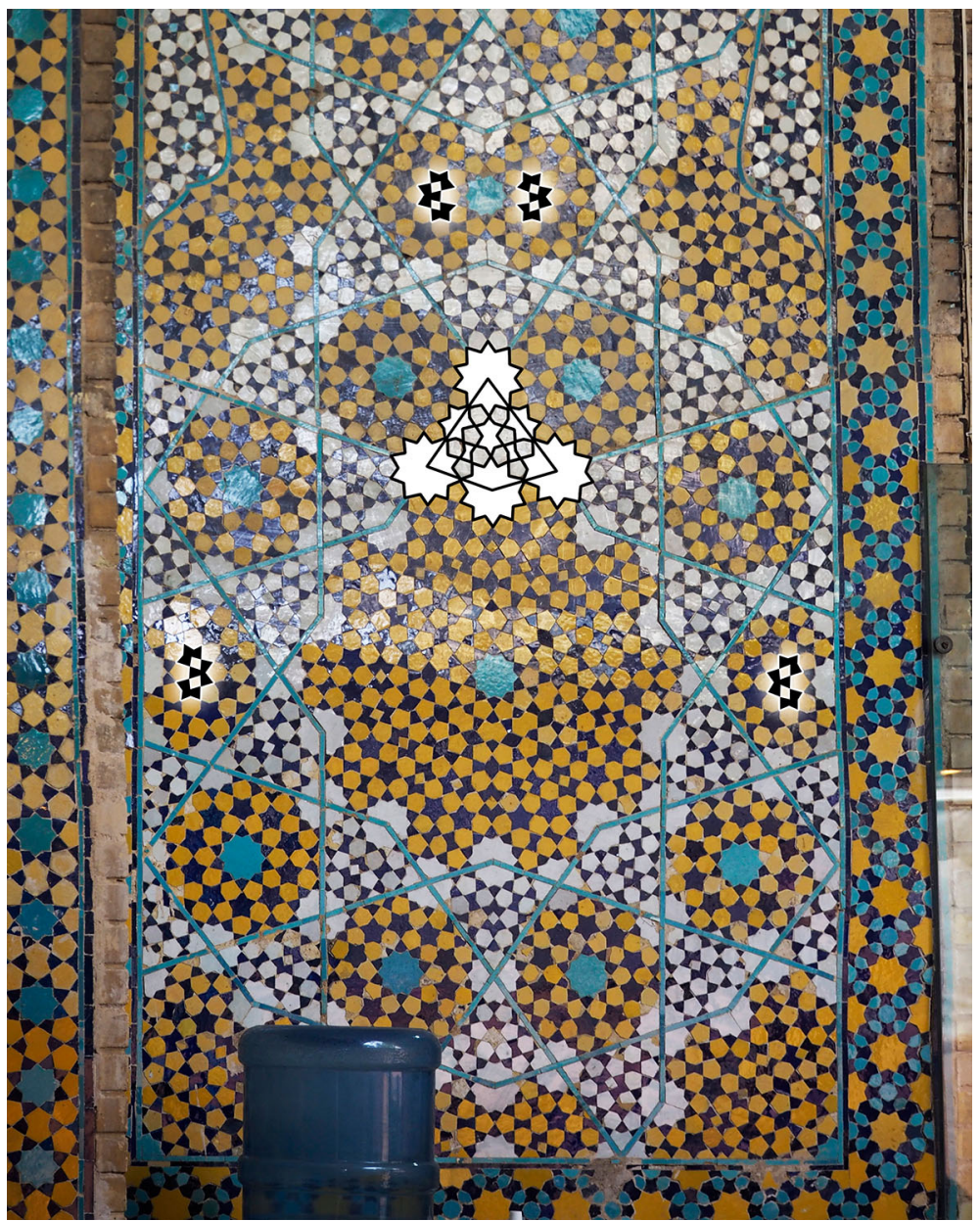

Fig. 58 Isfahan, Chahar Bagh Madrasa

We start from the simple idea to have a star centered on each crossing point (white dots in Fig. 62c) and also on each point of discontinuity (black dots). Now, we have to fix the width of the interlaces, and fill the shapes p1, p2, n1, n2, n3 and the three additive shapes of the interlaces, b1, b2, b3.

It makes sense to start from the smallest tile $\mathrm{n} 2$, every other will be inferred from it: In this story, small is beautiful, small is powerful!

The simplest solution consists in having connected stars along the small edge (Fig. 63a). This correspond to the mapping of N2 in our System 2 ("Two Self Similarity Systems on $\{\mathrm{S}\}$ "). Then we choose again the simplest solution for the tile b1 (Fig. 63b). Therefore we get the solution for each mapping of the three shapes of the interlaces (Fig. 63c). In fact, we can now get rid of all secondary shapes and consider only the mapping of N2, with the lines of the interlaces inside (Fig. 63d). 
Fig. 59 Isfahan, Friday mosque

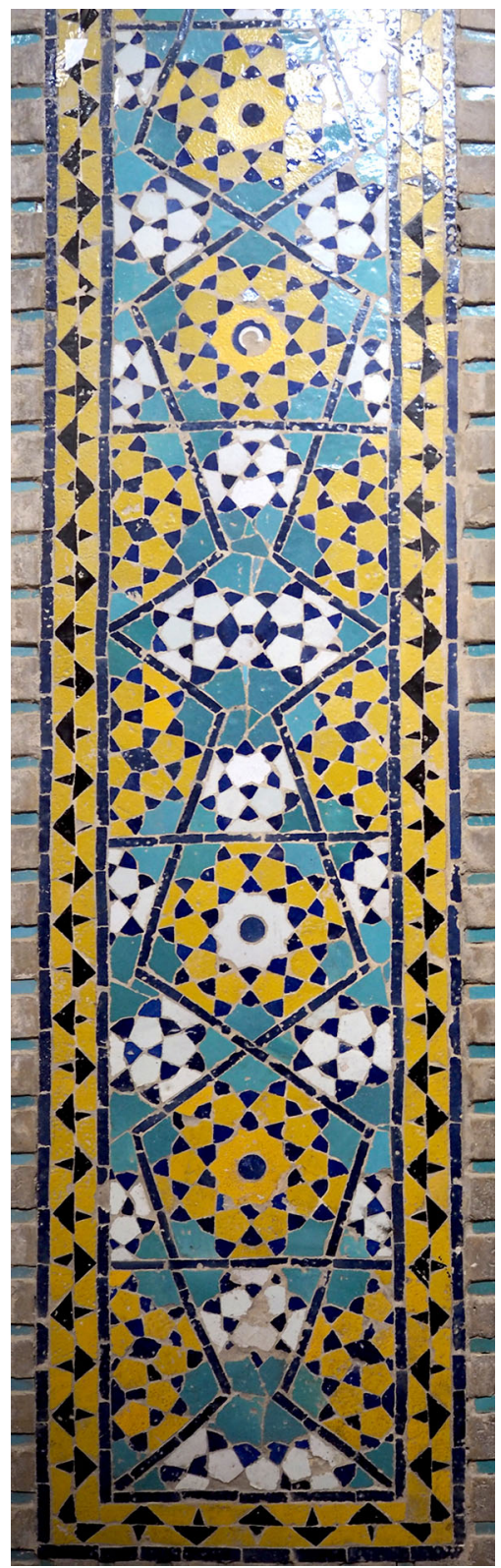




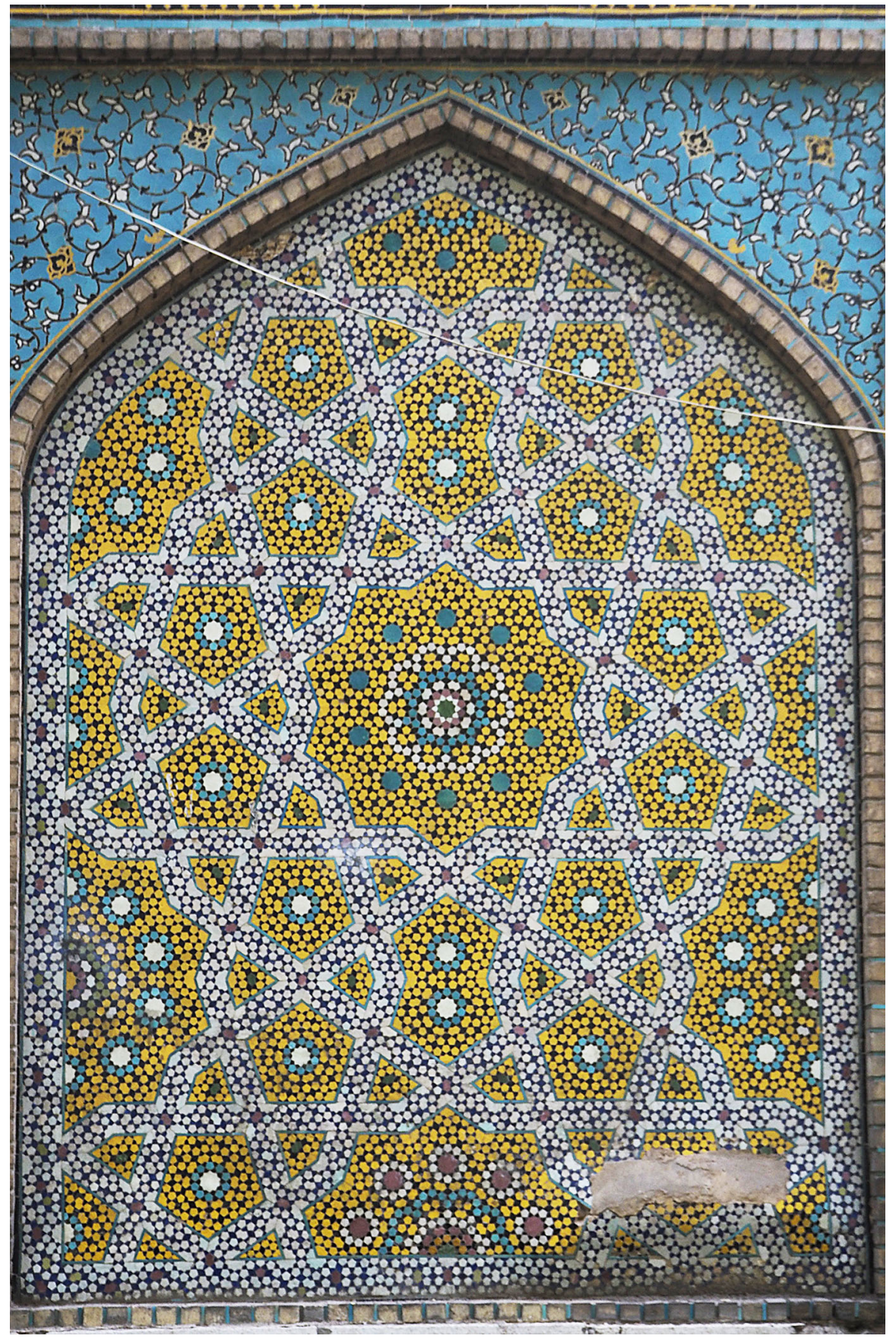

Fig. 60 Isfahan, Chahar Bagh Madrasa 


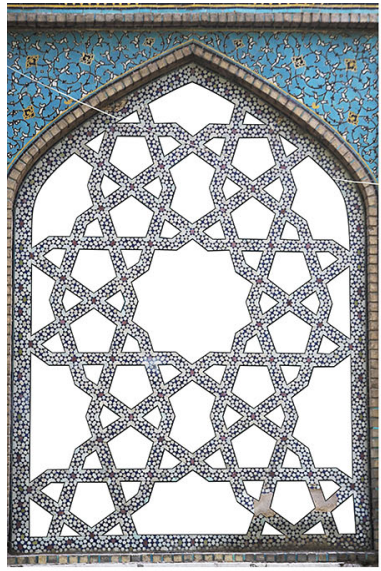

a

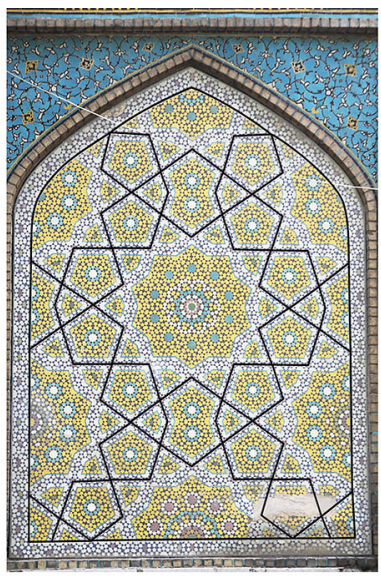

b

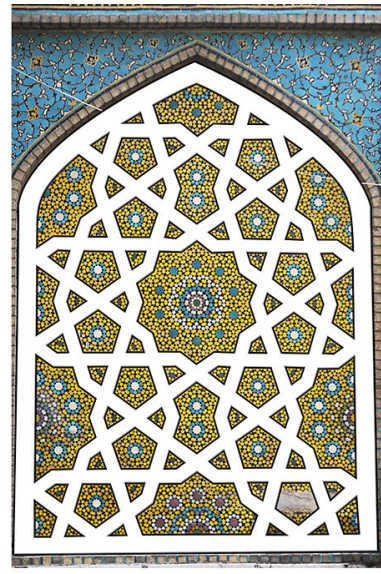

c

Fig. 61 Middle the basic "Mother of Tiling" pattern. Left the decorated interlaces, right the inside shapes

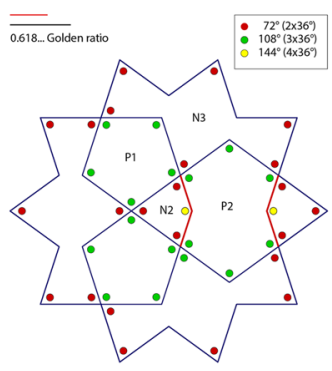

a

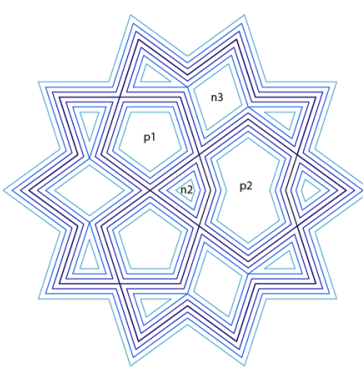

b

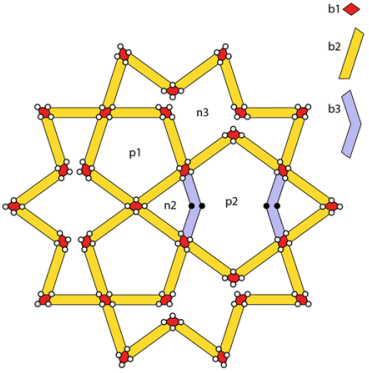

c

Fig. 62 The tiles of a Kond pattern (a). Adding interlaces of different width (b). Components of a pattern with interlaces $(\mathbf{c})$

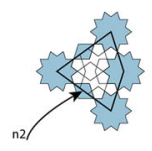

a

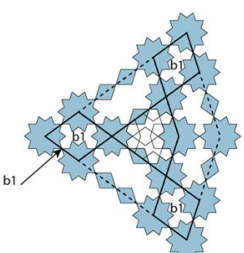

b

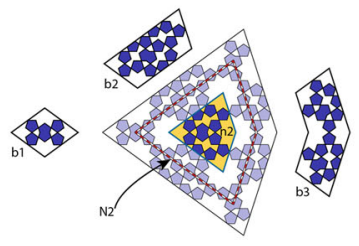

c

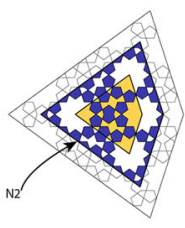

d

Fig. 63 From n2 to N2, via the interlaces 


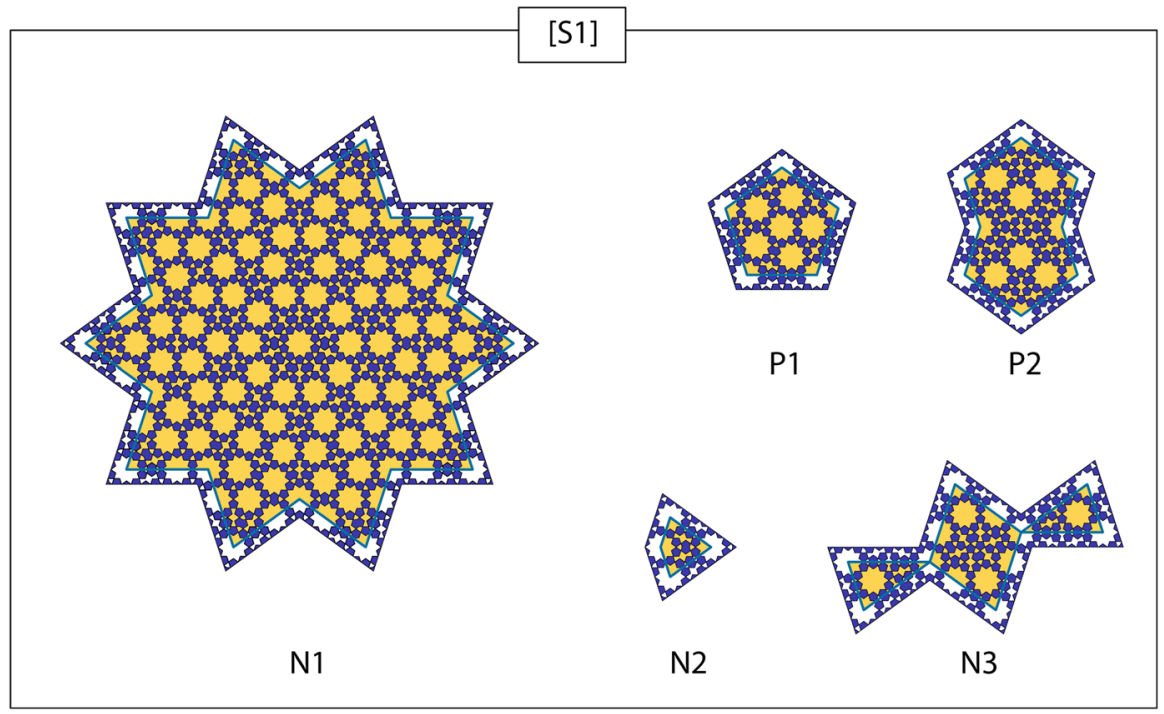

Fig. 64 The set of mapped tiles of the self-similar system with interlaces. There is again two positive tiles, P1 and P2, and three negatives, N1, N2 and N3

Then, we infer the mapping of the other tiles P1, P2, N1, N3 after drawing the half interlaces along their perimeter. Fortunately there is a solution, even for the tile N3 (Fig. 64), for which the length of the interlaces is maximal.

Notice that, contrary to self-similar systems 1 and 2, there is nothing special for the Sormedan N3.

Figure 65 shows a detail of a 3-level pattern made from this system, which is a generalization of the Chahar Bagh tiling. We have used color inversion to highlight the interlaces of level 1 and 2.

\section{Conclusion}

In my experience, it is often useful to experiment with alternative views on geometric patterns... or anything. Sometimes this can open new paths, provoke surprising connections, and stimulate creativity.

This work was starting from very simple questions. Then, on the way, one thing comes out and leads to another thing, and so on... Working on variations is a neverending process!

So, more have to be done. About the traditional heritage, new points of view will certainly emerge in the future. Concerning pattern creation... Space is open.

In everything, my interest does not goes to the thing itself, but to its relationships with other things. 


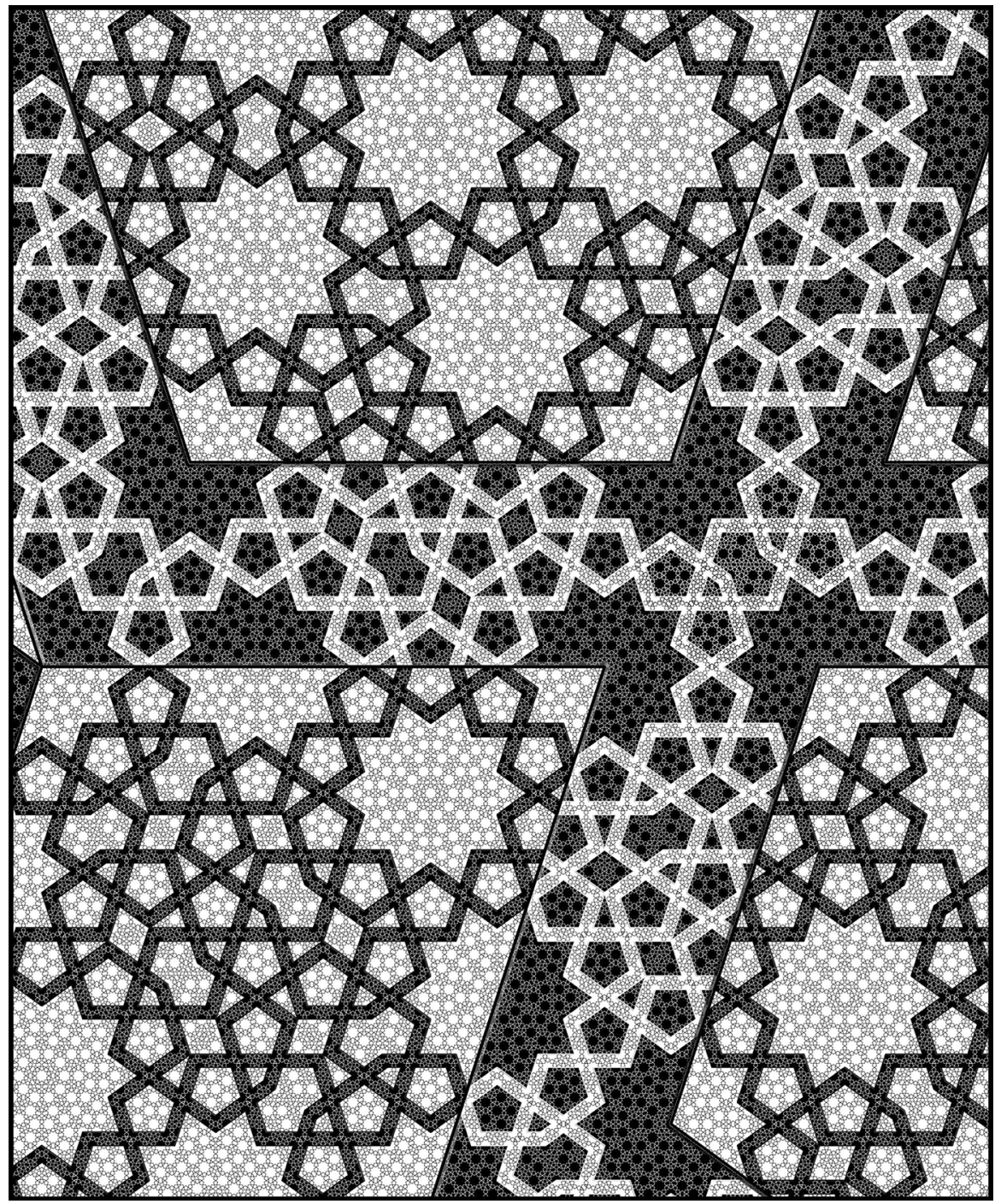

Fig. 65 Three levels of a self similar pattern with interlaces (detail)

\section{References}

J. Bonner, "Three traditions of self-similarity in fourteenth and fifteenth century Islamic geometric ornament", Proc. ISAMA/Bridges, (Granada, 2003), eds. R. Sarhangi and N. Friedman, pp. 1-12, 2003.

J. Bonner, "The Historical Use of Polygonal System to Create Islamic Geometric Patterns", Les tracés de l'Arabesque géométrique", Académie des Arts Traditionnels, Casablanca, 2013

J.-M. Castera, "Flying Patterns", Proc. ISAMA/Bridges, Coimbra, 2011. Can be downloaded from Bridge's web site, or from http://castera.net/entrelacs/public/articles/Flying_Patterns.pdf 
J.-M. Castera, "Arabesques for Abu-Dhabi”, Symmetry: Culture and Science, Vol. 19, Numbers 2-3, 165-174, 2008.

J.-M. Castera, Arabesques: Art Décoratif au Maroc, ACR Edition, 1996.

P. R. Cromwell, "Modularity and hierarchy in Persian geometric ornament", preprint 2013.

P. R. Cromwell, "The search for quasi-periodicity in Islamic 5-fold ornament", Math. Intelligencer 31 no $136-56,2009$

E. H. Hankin, "The Drawing of Geometric Patterns in Saracenic Art", Memories of the Archaeological Society of India, Vol 15, Government of India, 1925.

C. S. Kaplan, "Islamic star patterns from polygons in contact", Graphics Interface 2005, ACM International Conference Proceeding Series 112, pp. 177-186, 2005

C. S. Kaplan, Computer Graphics and Geometric Ornamental Design, PhD thesis, 2002. Can be downloaded on http://www.cgl.uwaterloo.ca/ csk/phd/

A. J. Lee, "Islamic star patterns", Muqarnas IV: An Annual on Islamic Art and Architecture, ed. O. Grabar, Leiden, pp. 182-197, 1987.

P. Lu and P.J. Steinhardt, "Decagonal and quasi-crystalline tilings in medieval Islamic architecture", Science 315, pp. 1106-1110, 2007

E. Makovicky, "800-year old pentagonal tiling from Maragha, Iran, and the new varieties of aperiodic tiling it inspired", Fivefold Symmetry, ed. I. Hargittai, World Scientific, pp. 67-86, 1992.

H. Mofid, M. Raieszadeh, Revival of the Forgotten Arts: Principles of the Traditional Architecture in Iran According to Hossein Lorzadeh, Mola Publications, Tehran, Iran, 1995 (In Persian)

G. Necipogglu, The TopkapiScroll: Geometry and Ornament in Islamic Architecture, Getty sCenter Publication, 1995.

A. Paccard, Le Maroc et l'artisnat traditionnel Islamique dans l'architecture, Atelier 74, 1980

M. Pelletier, "Zellij Qusicrystals-A Gallery", Les tracés de l'Arabesque géométrique, Académie des Arts Traditionnels, Casablanca, 2013

R. Sarhangi, "Polyhedral Modularity in a Special Class of Decagram Based Interlocking Star Polygons", Bridges 2012: Mathematics, Music, Art, Architecture, Culture, pp. 165-174, 2012.

A. Shaarbaf, Ghirih and karbandi, Volume 1, The National Organization for Protection of Iran's Antiquities, 1982.

Jean-Marc Castera holds a degree in Mathematics. He created an experimental course on Mathematics and Arts at the university of Paris 8. He started working on traditional geometric art, published two books on the subject. In the meantime he left teaching and began working on 3D computer images and animations. The strong relationship between the arts of zellij, muqarnas and the 2Doctagonal quasicrystals (or Ammann Patterns) have been an important source of inspiration in his work as Pattern designer. Having worked a lot on traditional art, he is now exploring new paths for geometric arabesque, in continuity of its 'spirit' more than its shape. Lately, working in total freedom on projects with the English architect Norman Foster has been the perfect opportunity to experiment in this way. 